\title{
Hedonic Regression Models for Tokyo Condominium Sales
}

W. Erwin Diewert and Chihiro Shimizu, ${ }^{1}$

December 26, 2015

Discussion Paper 15-07,

School of Economics,

The University of British Columbia,

Vancouver, Canada, V6T 1 Z1.

\begin{abstract}
The paper fits a hedonic regression model to the sales of condominium units in Tokyo over the period 2000-2015. The problem is complicated by the need to decompose the selling price of a unit into a component that can be attributed to the structure area of the unit and another component that can be attributed to the unit's share of land value. There is very little information on the value of condominium land and so this paper develops a methodology for reducing this knowledge gap. The paper extends the builder's model which was developed in Eurostat (2013). Characteristics which prove to be important in explaining condominium prices are: the floor space area of the unit, the total land area of the building, the number of units in the building, the total number of stories in the building, the height of the sold unit, the age of the structure and the amount of excess land. The paper also derives an estimate for the annual geometric structure depreciation rate for condominiums in Tokyo.
\end{abstract}

\section{Key Words}

Condominium property price indexes, System of National Accounts, Balance Sheets, methods of depreciation, land and structure price indexes, hedonic regressions.

\section{Journal of Economic Literature Classification Numbers}

C2, C23, C43, E31, R21.

\footnotetext{
${ }^{1}$ W. Erwin Diewert: School of Economics, University of British Columbia, Vancouver B.C., Canada, V6T $1 Z 1$ and the School of Economics, University of New South Wales, Sydney, Australia (email: erwin.diewert@ubc.ca) and Chihiro Shimizu: Institute of Real Estate Studies, National University of Singapore (e-mail: cshimizu@nus.edu.sg) . The authors gratefully acknowledge financial support from the Australian Research Council (LP0884095) and the SSHRC of Canada. The authors thank Kevin Fox, Mick Silver and Nigel Stapledon for helpful comments.
} 


\section{Introduction}

The international System of National Accounts asks countries to provide estimates for the value of assets held by the various sectors in the economy. These estimates are supposed to appear in the Balance Sheet Accounts of the country. An important asset for the Household Sector is the stock of housing. For many modeling purposes, it is important to not only have estimates for the value of the housing stock but to decompose the overall value into (additive) land and structure components and then to further decompose these value aggregates into constant quality price and quantity components. ${ }^{2}$ This is not an easy task. When a housing property is sold, the selling price values the sum of the structure and land components and so a structure-land decomposition must be obtained by a modeling exercise. The problem of obtaining constant quality price components for the land and structure components of a housing unit is further complicated by the fact that housing units are almost always unique assets. A dwelling unit is different from any other dwelling unit at the same period in time due to its location, which is unique (and as locations vary for the same physical structure, the price of the land plot for the unit will generally change due to locational amenities). The same dwelling unit compared over space will also be different due to depreciation and possible renovations to the structure.

Our task in the present paper is to present a modeling strategy to provide a decomposition of condominium sales into constant quality price and quantity components for the structure and land components of the condo sale. We will follow roughly the same strategy as was outlined in Chapter 8 of Eurostat (2013) where a similar modeling strategy was applied to sales of detached dwellings. Our present task is much more difficult for two reasons:

- The value of a condominium unit is made up of a structure and a land component. But it is difficult to know exactly how to allocate the share of the total land value of the building plot to any particular unit. This problem does not arise for detached houses.

- There is much more heterogeneity in condo units than there is in detached dwelling units. With detached dwelling units, the suburb of the unit, its floor space area, the area of the land plot and the age of the unit can explain a great deal of the variation in detached houses. However, these variables are not sufficient to explain the variation in condo prices. As we shall see, other important explanatory variables are the height of the building, the height of the condo unit that is being sold, and the area of the land plot that is not being used to support the building.

Section 2 explains our quarterly data set which covers sales of condo units in Tokyo over the years 2000-2015.

Section 3 explains our basic regression model. We find that this preliminary regression model does not provide a reasonable decomposition of condo value into additive land and

\footnotetext{
${ }^{2}$ Governments in many countries impose separate tax rates on the land and structure components of residential properties. Thus if these taxes are to be based on market values, it is important to be able to determine the values of these land and structure components in a scientific way.
} 
structure components, which is required for national income accounting purposes. Thus we construct an estimated imputed structure value for the condo unit and subtract this imputed value from the selling price of the condo unit to obtain an imputed land value that can be associated with the condo unit. In sections 4-10, we use these imputed land values as the dependent variable in our regression models in an attempt to find characteristics which can explain the variation in these imputed land prices. In section 11, we return to the actual selling prices for the condo units as the dependent variable in our regression model, using the land characteristics that we discovered were useful explanatory variables for the regressions in sections 4-10. In section 11, we now estimate the annual structure geometric depreciation rate instead of assuming it. Section 12 introduces a few additional characteristics into the regression model; these characteristics are thought to affect the structure value rather than the land value component of the total value of the condo unit.

In section 13, we group the 9 wards of Tokyo for which we have data into rich, medium and poorer wards and estimate ward time dummy variables for each type of ward. However, as will be shown in section 14, the resulting ward specific land prices turned out to be too variable to be credible. The basic problem is that we do not have a large enough number of observations to support the model presented in section 14. However, it is useful to show how our model can provide more detailed land prices by local area, if adequate data were available.

Section 14 shows how the separate land prices generated by the models in sections 12 and 13 can be combined with our structure prices to generate overall condo price indexes. The results presented in this section lead us to prefer the model presented in section 12 over the model presented in section 13.

Section 15 compares our preferred overall condo price index (generated by the model in section 12) to four other indexes. The first alternative index is an approximate price index for the stock of condo units in our 9 wards of Tokyo as opposed to our section 12 overall condo price index which is an index for the sales of condo units in the 61 quarters in our sample. However, we show that the two indexes are virtually identical. The next two alternative indexes are simple indexes based on the mean and median values of sales of condo units in the 61 quarters. These indexes perform poorly due to their variability and downward biases (due to their neglect of depreciation). Our final comparison index is based on a simple traditional time dummy hedonic regression. The resulting time dummy based index performs quite well in that it is close to our preferred indexes. ${ }^{3}$

Section 16 concludes.

\section{The Tokyo Condominium Data}

Our basic data set is on sales of condominium units located in 9 Wards in the central area of Tokyo over the 61 quarters starting at the first quarter of 2000 and ending at the first

\footnotetext{
${ }^{3}$ However, the time dummy approach does not generate separate land and structure price components, which is the main purpose of our paper.
} 
quarter of 2015. In addition to the sales prices, various characteristics of the properties were obtained from the website, Suumo (Residential Information Website), provided by Recruit Co., Ltd., one of the largest vendors of residential listings information in Japan. This source provides time series of listed prices from the week when it is first posted until the week it is removed due to its sale. ${ }^{4}$ We used the price in the final week because this can be safely regarded as sufficiently close to the contract price. ${ }^{5}$

There were a total of 3232 observations (after range deletions) in our sample of sales of condo units in Tokyo. ${ }^{6}$ The definitions for the selling price and 11 characteristics of the units sold and their units of measurement are as follows:

$\mathrm{V}=$ The value of the sale of the condo unit in $10,000 \mathrm{Yen}^{7}$

$\mathrm{S}=$ Structure area (floor space area) of the condo in units of meters squared;

$\mathrm{TS}=$ Floor space area for the entire building;

$\mathrm{TL}=$ Lot area for the entire structure in units of meters squared;

$\mathrm{A}=$ Age of the structure in years;

$\mathrm{H}=$ The story of the unit; i.e., the height of the unit that was sold;

$\mathrm{TH}=$ The total number of stories in the building; i.e., the total height of the building;

$\mathrm{NB}=$ Number of bedrooms in the unit;

TW $=$ Walking time in minutes to the nearest subway station;

$\mathrm{TT}=$ Subway running time in minutes to the Tokyo station from the nearest station during the day (not early morning or night);

$\mathrm{SCR}=$ Reinforced concrete construction dummy variable (= 1 if reinforced; 0 otherwise);

SOUTH=Dummy variable ( $=1$ if the unit faces south; 0 otherwise).

After range trimming, the minimum and maximum values for the various variables are listed in Table 1. It can be seen that even after trimming, there is a considerable amount of variation left in the data. ${ }^{8}$

\footnotetext{
${ }^{4}$ There are two reasons for the listing of a unit being removed from the magazine: a successful deal or a withdrawal (i.e. the seller gives up looking for a buyer and thus withdraws the listing). We were allowed access to information regarding which the two reasons applied for individual cases and we discarded those transactions where the seller withdrew the listing.

${ }^{5}$ Recruit Co., Ltd. provided us with information on contract prices for about 24 percent of all listings. Using this information, we were able to confirm that prices in the final week were almost always identical with the contract prices; see Shimizu, Nishimura and Watanabe (2012).

${ }^{6}$ It is risky to estimate hedonic regression models over wide ranges when observations are sparse at the beginning and end of the range of each variable. Moreover, real estate data usually contains many outliers and trimming the range of the independent variables will typically help eliminate some outliers.

${ }^{7}$ The variable $V$ is $V_{t n}$ where $t=1, \ldots, 64$ indicates the quarter when the unit was sold and $n=1 \ldots, N(t)$ indicates the nth condo sale in quarter $\mathrm{t}$ and $\mathrm{N}(\mathrm{t})=$ the total number of condo sales in our sample during quarter $\mathrm{t}$.

${ }^{8}$ Table 1 also reflects the results of range trimming for three synthetic variables: (i) $\mathrm{L}_{\mathrm{S}} \equiv(\mathrm{S} / \mathrm{TS}) \mathrm{TL}$ (this is an imputation for the share of the property's total land area TL that can be attributed to the sold unit where the unit has floor space area $\mathrm{S}$ and the building has total floor space area TS); (ii) the footprint ratio $\mathrm{F}_{\mathrm{R}}$ of the structure which is equal to the ratio of the land area occupied by the structure (TS/TH) to the total property land area TL so $F_{R} \equiv(T S / T H) / T L$ and (iii) an approximation to the useable floor space ratio of the building, UFSR $\equiv(\mathrm{N} \times \mathrm{S}) / \mathrm{TS}$ where $\mathrm{N}$ is the number of units in the building, $\mathrm{S}$ is the floor space of the sold unit and TS is the total floor space of the building, including common space. We deleted observations that fell outside the following range limits: $7 \leq \mathrm{L}_{\mathrm{S}} \leq 60 ; 0.1 \leq \mathrm{F}_{\mathrm{R}} \leq 0.8$ and $0.5 \leq \mathrm{UFSR} \leq 1.5$.
} 
Table 1: Descriptive Statistics for the Variables

\begin{tabular}{|c|c|c|c|c|c|}
\hline Name & No. of Obs. & Mean & Std. Dev & Minimum & Maximum \\
\hline $\mathbf{V}$ & 3232 & 2594.3 & 730.18 & 1080.0 & 6200.0 \\
\hline $\mathbf{S}$ & 3232 & 64.017 & 9.8155 & 40.00 & 99.68 \\
\hline TS & 3232 & 3859.7 & 2165.2 & 569.44 & 12857.0 \\
\hline TL & 3232 & 1355.5 & 790.30 & 153.64 & 3460.2 \\
\hline $\mathbf{A}$ & 3232 & 14.926 & 7.9680 & 1.00 & 34.42 \\
\hline $\mathbf{H}$ & 3232 & 5.6364 & 6.2438 & 3 & 19 \\
\hline TH & 3232 & 8.9465 & 3.0736 & 3 & 22 \\
\hline NB & 3232 & 2.7024 & 0.53813 & 2 & 5 \\
\hline TW & 3232 & 10.601 & 4.9076 & 1 & 19 \\
\hline TT & 3232 & 28.087 & 8.5236 & 12.1 & 48.0 \\
\hline SRC & 3232 & 0.37655 & 0.48459 & $\mathbf{0}$ & 1 \\
\hline SOUTH & 3232 & 0.39882 & 0.48973 & $\mathbf{0}$ & 1 \\
\hline
\end{tabular}

In addition to the above variables, we also have information on which Ward of Tokyo the sales took place. We used this information to create ward dummy variables, $\mathrm{D}_{\mathrm{W}, \mathrm{tn}, \mathrm{j}}$, which will be described more fully later. The 9 Wards for which we have data are as follows: Ward 1 = Sumida; Ward 2 = Koto; Ward 3 = Kita; Ward 4 = Arakawa; Ward $5=$ Itabashi; Ward 6= Nerima; Ward $7=$ Adachi; Ward $8=$ Katsushika and Ward $9=$ Edogawa.

In order to reduce multicollinearity between the various independent variables listed above (and to achieve consistency with national accounts data), ${ }^{9}$ we will assume that the value of a new structure in any quarter is proportional to a Construction Cost Price Index for Tokyo. ${ }^{10}$ We denote the value of this index during quarter $\mathrm{t}$ as $\mathrm{p}_{\mathrm{St} .}{ }^{11}$

\section{The Basic Builder's Model}

The builder's model for valuing a residential property postulates that the value of a residential property is the sum of two components: the value of the land which the structure sits on plus the value of the residential structure.

In order to justify the model, consider a property developer who builds a structure on a particular property. The total cost of the property after the structure is completed will be equal to the floor space area of the structure, say S square meters, times the building cost per square meter, $\beta_{\mathrm{t}}$ during quarter $\mathrm{t}$, plus the cost of the land, which will be equal to the cost per square meter, $\alpha_{t}$ during quarter $t$, times the area of the land site, L. Now think of a sample of properties of the same general type, which have prices or values $V_{t n}$ in period

\footnotetext{
${ }^{9}$ See Diewert, de Haan and Hendriks (2011) (2015) for evidence on this multicollinearity problem in the context of residential detached housing data using Dutch data.

${ }^{10}$ This index was constructed by the Construction Price Research Association which is now an independent agency but prior to 2012 was part of the Ministry of Land, Infrastructure, Transport and Tourism (MLIT), a ministry of the Government of Japan.

${ }^{11}$ In quarter $t, p_{S t}$ is equal to the average cost of construction of a condo unit per square meter for that quarter in units of 10,000 yen. This is another exogenous series that is required in order to implement our hedonic regression model.
} 
$\mathrm{t}^{12}$ and structure areas $\mathrm{S}_{\mathrm{tn}}$ and land areas $\mathrm{L}_{\mathrm{tn}}$ for $\mathrm{n}=1, \ldots, \mathrm{N}(\mathrm{t})$ where $\mathrm{N}(\mathrm{t})$ is the number of observations in period $\mathrm{t}$. Assume that these prices are equal to the sum of the land and structure costs plus error terms $\varepsilon_{\text {tn }}$ which we assume are independently normally distributed with zero means and constant variances. This leads to the following hedonic regression model for period $t$ where the $\alpha_{t}$ and $\beta_{t}$ are the parameters to be estimated in the regression: ${ }^{13}$

(1) $\mathrm{V}_{\mathrm{tn}}=\alpha_{\mathrm{t}} \mathrm{L}_{\mathrm{tn}}+\beta_{\mathrm{t}} \mathrm{S}_{\mathrm{tn}}+\varepsilon_{\mathrm{tn}}$;

$\mathrm{t}=1, \ldots, 61 ; \mathrm{n}=1, \ldots, \mathrm{N}(\mathrm{t})$

Note that the two characteristics in our simple model are the quantities of land $\mathrm{L}_{\text {tn }}$ and the quantities of structure floor space $S_{t n}$ associated with property $n$ in period $t$ and the two constant quality prices in period $t$ are the price of a square meter of land $\alpha_{t}$ and the price of a square meter of structure floor space $\beta_{\mathrm{t}}$.

The hedonic regression model defined by (1) applies to new structures. But it is likely that a model that is similar to (1) applies to older structures as well. Older structures will be worth less than newer structures due to the depreciation of the structure. Assuming that we have information on the age of the structure $n$ at time $t$, say $A(t, n)$, and assuming a geometric (or declining balance) depreciation model, a more realistic hedonic regression model than that defined by (1) above is the following basic builder's model: ${ }^{14}$

(2) $V_{t n}=\alpha_{t} L_{t n}+\beta_{t}\left(1-\delta_{t}\right)^{A(t, n)} S_{t n}+\varepsilon_{t n}$;

$$
\mathrm{t}=1, \ldots, 61 ; \mathrm{n}=1, \ldots, \mathrm{N}(\mathrm{t})
$$

where the parameter $\delta_{\mathrm{t}}$ reflects the net geometric depreciation rate as the structure ages one additional period. Thus if the age of the structure is measured in years, we would expect an annual net depreciation rate to be between 1.0 and $4.0 \% .{ }^{15}$ Note that $(2)$ is now a nonlinear regression model whereas (1) was a simple linear regression model. The period t constant quality price of land will the estimated coefficient for the parameter $\alpha_{t}$ and the price of a unit of a newly built structure for period t will be the estimate for $\beta_{t}$. The period $t$ quantity of land for condo unit $n$ is $L_{t n}$ and the period $t$ quantity of structure

\footnotetext{
${ }^{12}$ The period index $\mathrm{t}$ runs from 1 to 61 where period 1 corresponds to $\mathrm{Q} 1$ of 2000 and period 61 corresponds to Q1 of 2015.

${ }^{13}$ Other papers that have suggested hedonic regression models that lead to additive decompositions of property values into land and structure components include Clapp (1980; 257-258), Bostic, Longhofer and Redfearn (2007; 184), Diewert (2007; 19-22) (2010), Francke (2008; 167), Koev and Santos Silva (2008), Eurostat (2013; 94-99), Rambaldi, McAllister, Collins and Fletcher (2010), Diewert, Haan and Hendriks (2011) (2015) and Diewert and Shimizu (2015) (2016).

${ }^{14}$ This formulation follows that of Diewert (2010), Diewert, Haan and Hendriks (2015), Eurostat (2013) and Diewert and Shimizu (2015) (2016) in assuming property value is the sum of land and structure components but movements in the price of structures are proportional to an exogenous structure price index. This formulation is designed to be useful for national income accountants who require a decomposition of property value into structure and land components. They also need the structure index which in the hedonic regression model to be consistent with the structure price index they use to construct structure capital stocks. Thus the builder's model is particularly suited to national accounts purposes.

${ }^{15}$ This estimate of depreciation is regarded as a net depreciation rate because it is equal to a "true" gross structure depreciation rate less an average renovations appreciation rate. Since we do not have information on renovations and major repairs to a structure, our age variable will only pick up average gross depreciation less average real renovation expenditures.
} 
for condo unit $n$, expressed in equivalent units of a new structure, is $\left(1-\delta_{t}\right)^{A(t, n)} S_{t n}$ where $\mathrm{S}_{\mathrm{tn}}$ is the floor space area of condo unit $\mathrm{n}$ in period $\mathrm{t}$.

Note that the above model is a supply side model as opposed to the demand side model of Muth (1971) and McMillen (2003). Basically, we are assuming competitive suppliers of condominium units so that initially, ${ }^{16}$ we are in Rosen's $(1974 ; 44)$ Case (a), where the hedonic surface identifies the structure of supply. This assumption is justified for the case of newly built condos but it is less well justified for sales of existing condo units.

There are at least two major problems with the hedonic regression model defined by (2):

- The multicollinearity problem and

- The problem of imputing an appropriate share of the total land area to a particular condominium unit.

Experience has shown that it is usually not possible to estimate sensible land and structure prices in a hedonic regression like that defined by (2) due to the multicollinearity between lot size and structure size. ${ }^{17}$ Thus in order to deal with the multicollinearity problem, we draw on exogenous information on the cost of building new condominium units from the Japanese Ministry of Land, Infrastructure, Transport and Tourism (MLIT) and we assume that the price of new structures is proportional to an official index of condominium building costs, $\mathrm{p}_{\mathrm{St}}$. Thus we replace $\beta_{\mathrm{t}}$ in (2) by $\beta \mathrm{p}_{\mathrm{St}}$ for $\mathrm{t}=$ $1, \ldots, 61$. This reduces the number of free parameters in the model by 60 .

The second problem is that it is not appropriate to allocate the entire land value of the condominium property to any particular unit that is sold in period t. Thus each condo unit in the building should be allocated a share of the total land value of the property. The problem is: how exactly should this imputed land share be calculated? There are two simple methods for constructing an appropriate land share: (i) Use the unit's share of floor space to total structure floor space or (ii) simply use $1 / \mathrm{N}$ as the share where $\mathrm{N}$ is the total number of units in the building. Thus define the following two land imputations for unit $\mathrm{n}$ in period $\mathrm{t}$ :

(3) $\mathrm{L}_{\mathrm{Stn}} \equiv\left(\mathrm{S}_{\mathrm{tn}} / \mathrm{TS}_{\mathrm{tn}}\right) \mathrm{TL}_{\mathrm{tn}} ; \mathrm{L}_{\mathrm{Ntn}} \equiv\left(1 / \mathrm{N}_{\mathrm{tn}}\right) \mathrm{TL}_{\mathrm{tn}}$;

$\mathrm{t}=1, \ldots, 61 ; \mathrm{n}=1, \ldots, \mathrm{N}(\mathrm{t})$

where $\mathrm{S}_{\mathrm{tn}}$ is the floor space area of unit $\mathrm{n}$ in period $\mathrm{t}, \mathrm{TS}_{\mathrm{tn}}$ is the total building floor space area, $\mathrm{TL}_{\mathrm{tn}}$ is the total land area of the building and $\mathrm{N}_{\mathrm{tn}}$ is the total number of units in the building for unit $\mathrm{n}$ sold in period $\mathrm{t}$. The first method of land share imputation is used by the Japanese land tax authorities. The second method of imputation implicitly assumes that each unit can enjoy the use of the entire land area and so an equal share of land for each unit seems "fair".

\footnotetext{
${ }^{16}$ In later sections of the paper, we will see that purchasers of condo units also influence the price of a condo unit.

${ }^{17}$ See Schwann (1998) and Diewert, de Haan and Hendriks (2011) and (2015) on the multicollinearity problem.
} 
We note that there is a problem with the first definition in (3). In order to allocate land across the $\mathrm{N}$ units in a building, the unit shares should add up to one. However, the shares $\mathrm{S}_{\mathrm{tn}} / \mathrm{TS}_{\mathrm{tn}}$, if available for every unit in the building, would add up to a number less than one because the unit floor space areas, $S_{\mathrm{tn}}$, if summed over all units in the building add up to privately owned floor space which is less than total building floor space $\mathrm{TS}_{\mathrm{tn}}$. Total building floor space includes halls, elevators, storage space, furnace rooms and other "public" floor space. Unfortunately, our data set does not have the sum of privately owned floor space in the building so we will use the first definition in (3) as an approximation to the unit's land share.

We can calculate an approximation to total building privately owned floor space for observation $\mathrm{n}$ in period $\mathrm{t}$ as $\mathrm{N}_{\mathrm{tn}} \mathrm{S}_{\mathrm{tn}}$. Thus an imperfect estimate of the ratio of privately owned floor space to total floor space for unit $\mathrm{n}$ in period $\mathrm{t}$ is $\mathrm{N}_{\mathrm{tn}} \mathrm{S}_{\mathrm{tn}} / \mathrm{TS}_{\mathrm{tn}}$. The sample wide average of these ratios was 0.899 . Thus to account for shared structure space, we replaced the owned floor space variable in (2), $\mathrm{S}_{\mathrm{tn}}$, by (1/0.899) $\mathrm{S}_{\mathrm{tn}}=(1.1) \mathrm{S}_{\mathrm{tn}}$.

In order to get preliminary land price estimates, we substituted the land estimates defined by (3) into the regression model (2), we replaced the $\beta_{\mathrm{t}}$ by $\beta \mathrm{p}_{\mathrm{St}}$, the $\mathrm{S}_{\mathrm{tn}}$ by (1.1) $\mathrm{S}_{\mathrm{tn}}$ and we assumed that the annual geometric depreciation rate $\delta_{t}$ was equal to 0.03 . The resulting linear regression models become the models defined by (4) and (5) below:

(4) $\mathrm{V}_{\mathrm{tn}}=\alpha_{\mathrm{t}} \mathrm{L}_{\mathrm{Stn}}+(1.1) \beta \mathrm{p}_{\mathrm{St}}(1-0.03)^{\mathrm{A}(\mathrm{t}, \mathrm{n})} \mathrm{S}_{\mathrm{tn}}+\varepsilon_{\mathrm{tn}}$;

(5) $\mathrm{V}_{\mathrm{tn}}=\alpha_{\mathrm{t}} \mathrm{L}_{\mathrm{Ntn}}+(1.1) \beta \mathrm{p}_{\mathrm{St}}(1-0.03)^{\mathrm{A}(\mathrm{t}, \mathrm{n})} \mathrm{S}_{\mathrm{tn}}+\varepsilon_{\mathrm{tn}}$;

$\mathrm{t}=1, \ldots, 61 ; \mathrm{n}=1, \ldots, \mathrm{N}(\mathrm{t})$

$\mathrm{t}=1, \ldots, 61 ; \mathrm{n}=1, \ldots, \mathrm{N}(\mathrm{t})$.

Thus we have 3232 degrees of freedom to estimate 61 land price parameters $\alpha_{t}$ and one structure quality parameter $\beta$ for a total of 62 parameters for each of the models defined by (4) and (5).

The $\mathrm{R}^{2}$ for the models defined by (4) and (5) were only 0.5894 and $0.5863,{ }^{18}$ which was not entirely satisfactory. Both models have approximately the same fit and generate similar estimates for the structure quality parameter $\beta$. The estimates for $\beta$ were 2.164 and 2.154 respectively which was totally unsatisfactory because these parameters should have been close to unity. Moreover the land price indexes that these regression models generated were subject to excessive volatility (due to the very high estimates for the structure quality parameter, $\beta$ ).

In order to deal with the problem of too high estimates of $\beta$, we decided not to estimate it. Moreover, we temporarily put aside the problem of jointly determining land and structure value to concentrate on determining sensible constant quality land prices. Once sensible land prices have been determined, we will then return to the problem of simultaneously determining land and structure values and constant quality price indexes.

\footnotetext{
${ }^{18}$ All of the $\mathrm{R}^{2}$ reported in this paper are equal to the square of the correlation coefficient between the dependent variable in the regression and the corresponding predicted variable. The log likelihood for the two models were -24739.6 and -24736.4 so both land imputation methods gave very similar results.
} 
Thus in the following sections 4-10, we will assume that the structure value for unit $\mathrm{n}$ in period $t, V_{\text {Stn }}$, is defined as follows:

(6) $\mathrm{V}_{\mathrm{Stn}} \equiv(1.1) \mathrm{p}_{\mathrm{St}}(1-0.03)^{\mathrm{A}(\mathrm{t}, \mathrm{n})} \mathrm{S}_{\mathrm{tn}}$;

$\mathrm{t}=1, \ldots, 61 ; \mathrm{n}=1, \ldots, \mathrm{N}(\mathrm{t})$.

Once the imputed value of the structure has been defined by (6), we define the imputed land value for condo $\mathrm{n}$ in period $\mathrm{t}, \mathrm{V}_{\mathrm{Ltn}}$, by subtracting the imputed structure value from the total value of the condo unit, which is $\mathrm{V}_{\mathrm{tn}}$ :

(7) $V_{L t n} \equiv V_{\text {tn }}-V_{S t n}$;

$\mathrm{t}=1, \ldots, 61 ; \mathrm{n}=1, \ldots, \mathrm{N}(\mathrm{t})$.

Thus in the following 7 sections, we will use $V_{L t n}$ as our dependent variable and we will attempt to explain variations in these imputed land values in terms of the property characteristics.

\section{The Introduction of Ward Dummy Variables}

For now, we will use the first land measure in (3) as our estimate of the share of total land that is imputed to unit $\mathrm{n}$ sold in period t; i.e., unit n's share of land in period $\mathrm{t}$ is measured as $\mathrm{L}_{\mathrm{Stn}}=\left(\mathrm{S}_{\mathrm{tn}} / \mathrm{TS}_{\mathrm{tn}}\right) \mathrm{TL}_{\mathrm{tn}}$. To start off, we will estimate the linear regression that is the pure land counterpart to (4); i.e., we will estimate the following linear regression model where imputed land value $\mathrm{V}_{\mathrm{Ltn}}$ has replaced total value $\mathrm{V}_{\mathrm{tn}}$ as the dependent variable:

(8) $\mathrm{V}_{\mathrm{Ltn}}=\alpha_{\mathrm{t}} \mathrm{L}_{\mathrm{Stn}}+\varepsilon_{\mathrm{tn}}$;

$\mathrm{t}=1, \ldots, 61 ; \mathrm{n}=1, \ldots, \mathrm{N}(\mathrm{t})$.

The above simple linear regression model has 61 land price parameters $\alpha_{t}$ to be estimated. The $\mathrm{R}^{2}$ between the observed and predicted variables was only 0.0064 and the $\log$ likelihood was -25913.6. These results are hardly stellar but on a positive note, the resulting land price index was reasonably behaved.

In order to take into account possible neighbourhood effects on the price of land, we introduce ward dummy variables, $\mathrm{D}_{\mathrm{W}, \mathrm{tn}, \mathrm{j}}$, into the hedonic regression (8). These 9 dummy variables are defined as follows: for $\mathrm{t}=1, \ldots, 61 ; \mathrm{n}=1, \ldots, \mathrm{N}(\mathrm{t}) ; \mathrm{j}=1, \ldots, 9:^{19}$

(9) $\mathrm{D}_{\mathrm{W}, \mathrm{tn}, \mathrm{j}} \equiv 1$ if observation $\mathrm{n}$ in period $\mathrm{t}$ is in Ward $\mathrm{j}$ of Tokyo;

$\equiv 0$ if observation $\mathrm{n}$ in period $\mathrm{t}$ is not in Ward $\mathrm{j}$ of Tokyo.

We now modify the model defined by (8) to allow the level of land prices to differ across the 9 Wards. The new nonlinear regression model is the following one:

(10) $\mathrm{V}_{\mathrm{Ltn}}=\alpha_{\mathrm{t}}\left(\sum_{\mathrm{j}=1}^{9} \omega_{\mathrm{j}} \mathrm{D}_{\mathrm{W}, \mathrm{tn}, \mathrm{j}}\right) \mathrm{L}_{\mathrm{Stn}}+\varepsilon_{\mathrm{tn}}$

$\mathrm{t}=1, \ldots, 61 ; \mathrm{n}=1, \ldots, \mathrm{N}(\mathrm{t})$.

\footnotetext{
${ }^{19}$ The sample average Ward selling prices for 8 Wards were as follows: 2746.2, 2748.1, 2893.2, 2493.7, $2585.8,2934.5,2174.2,2241.5,2814.6$. Thus there is a fair amount of variation in average selling prices across Wards.
} 
Comparing the models defined by equations (8) and (10), it can be seen that we have added an additional 9 ward relative land value parameters, $\omega_{1}, \ldots, \omega_{9}$, to the model defined by (8). However, looking at (9), it can be seen that the 61 land price parameters (the $\alpha_{t}$ ) and the 9 ward parameters (the $\omega_{\mathrm{j}}$ ) cannot all be identified. Thus we need to impose at least one identifying normalization on these parameters. We chose the following normalization:

(11) $\alpha_{1} \equiv 1$.

This normalization is convenient since the sequence of parameter estimates, the $\alpha_{t}$, will form a price index for condominium land in the 9 Wards where the index starts at unity. Taking into account the normalization (11), it can be seen that the model defined by (10) has 60 unknown land price parameters $\alpha_{\mathrm{t}}$ and 9 ward relative land price parameters $\omega_{\mathrm{j}}$. The regression model defined by (10) is now a nonlinear regression model. We estimated this model (and the subsequent nonlinear regression models) using the nonlinear regression option in Shazam; see White (2004). The $\mathrm{R}^{2}$ for this model turned out to be 0.1237 and the log likelihood (LL) was -25433.0 , a big increase of 480.6 over the LL of the model defined by (8). Thus the Ward variables are very significant determinants of Tokyo condominium land prices.

\section{The Introduction of Building Height as an Explanatory Variable}

It is likely that the height of the building increases the value of the land plot supporting the building, all else equal. In our sample of condo sales, the height of the building (the $\mathrm{TH}$ variable) ranged from 3 stories to 22 stories. However, there were very few observations for the last 7 height categories. ${ }^{20}$ Thus we collapsed the last seven height categories into a single category 14 and the remaining 13 height categories corresponded to building heights of 3 to 15 stories. Thus we define the building height dummy variables, $\mathrm{D}_{\mathrm{TH}, \mathrm{tn}, \mathrm{h}}$, as follows: $\mathrm{t}=1, \ldots, 61 ; \mathrm{n}=1, \ldots, \mathrm{N}(\mathrm{t}) ; \mathrm{h}=1, \ldots, 14$ :

(12) $\mathrm{D}_{\mathrm{TH}, \mathrm{tn}, \mathrm{h}} \equiv 1$ if observation $\mathrm{n}$ in period $\mathrm{t}$ is in building height category $\mathrm{h}$;

$\equiv 0$ if observation $\mathrm{n}$ in period $\mathrm{t}$ is not in building height category $\mathrm{h}$.

The new nonlinear regression model is the following one:
$\mathrm{V}_{\mathrm{Ltn}}=\alpha_{\mathrm{t}}\left(\sum_{\mathrm{j}=1}^{9} \omega_{\mathrm{j}} \mathrm{D}_{\mathrm{W}, \mathrm{tn}, \mathrm{j}}\right)\left(\sum_{\mathrm{h}=1}^{14} \chi_{\mathrm{h}} \mathrm{D}_{\mathrm{TH}, \mathrm{tn}, \mathrm{h}}\right) \mathrm{L}_{\mathrm{Stn}}+\varepsilon_{\mathrm{tn}}$
$\mathrm{t}=1, \ldots, 61 ; \mathrm{n}=1, \ldots, \mathrm{N}(\mathrm{t})$.

Comparing the models defined by equations (10) and (13), it can be seen that we have added an additional 14 building height parameters, $\chi_{1}, \ldots, \chi_{14}$, to the model defined by (10). However, looking at (13), it can be seen that the 61 land price parameters (the $\alpha_{t}$ ), the 9 ward parameters (the $\omega_{\mathrm{j}}$ ) and the 14 building height parameters (the $\chi_{\mathrm{h}}$ ) cannot all be identified. Thus we imposed the following identifying normalizations on these parameters:

\footnotetext{
${ }^{20}$ The number of observations for the last 7 height categories were as follows: $0,0,0,7,0,0,14$.
} 
(14) $\alpha_{1} \equiv 1 ; \chi_{1} \equiv 1$.

The $\mathrm{R}^{2}$ for this model turned out to be 0.2849 and the log likelihood was -24831.8 , a big increase of 601.2 over the LL of the model defined by (10) for the addition of 13 new parameters. Thus the height of the building is a very significant determinant of Tokyo condominium land prices. ${ }^{21}$

\section{The Introduction of the Height of the Unit as an Explanatory Variable}

The higher up a unit is, the better is the view on average and so we would expect the price of the unit would increase all else equal. The quality of the structure probably does not increase as the height of the unit increases so it seems reasonable to impute the height premium as an adjustment to the land price component of the unit.

We initially introduced the height of the unit (the $\mathrm{H}$ variable) as a categorical variable (like the height of the building in the previous section), but we found that this dummy variable approach could be replaced by using $\mathrm{H}$ as a continuous variable with little change in the fit of the model. Thus the new nonlinear regression model is the following one:

(15) $\mathrm{V}_{\mathrm{Ltn}}=\alpha_{\mathrm{t}}\left(\sum_{\mathrm{j}=1}^{9} \omega_{\mathrm{j}} \mathrm{D}_{\mathrm{W}, \mathrm{tn}, \mathrm{j}}\right)\left(\sum_{\mathrm{h}=1}^{14} \chi_{\mathrm{h}} \mathrm{D}_{\mathrm{TH}, \mathrm{tn}, \mathrm{h}}\right)\left(1+\gamma\left(\mathrm{H}_{\mathrm{tn}}-3\right)\right) \mathrm{L}_{\mathrm{Stn}}+\varepsilon_{\mathrm{tn}}$; $\mathrm{t}=1, \ldots, 61 ; \mathrm{n}=1, \ldots, \mathrm{N}(\mathrm{t})$.

Again, the normalizations (14) on the parameters in (15) were imposed. The lowest height for the units sold in our sample was $\mathrm{H}_{\mathrm{tn}}=3$. Thus for all the observations that correspond to the sold unit being located on the third floor of the building, the new parameter $\gamma$ in (15) will not affect the predicted value in the regression. However, for heights of the sold units that were greater than 3 , the regression implies that the land value will increase by $\gamma$ for each story that is above 3. The estimated value for $\gamma$ turned out to be $\gamma^{*}=0.0225(\mathrm{t}=6.44)$. Thus the imputed land value of a unit increases by $2.25 \%$ for each story above the threshold level of 3 . This is a reasonable number.

The $\mathrm{R}^{2}$ for this model turned out to be 0.2951 and the $\log$ likelihood was -24805.8 , a substantial increase of 26.0 over the LL of the model defined by (13) for the addition of one new parameter. Thus in addition to the height of the building, the height of the sold unit is also a very significant determinant of Tokyo condominium land prices.

\section{The Introduction of a More General Method of Land Imputation}

As was mentioned in Section 3 above, there are two simple methods for imputing the share of the building's total land area, $\mathrm{TL}_{\mathrm{tn}}$, to the sold unit. Up until now, we have used

\footnotetext{
${ }^{21}$ The sequence of estimated (except for $\chi_{1}$ ) height parameters, $\chi_{1}, \chi_{2} \ldots \chi_{14}$ (with $\mathrm{t}$ statistics in parentheses) is as follows: $1,1.098$ (11.9), 1.161 (13.5), 1.356 (13.6), 1.4329 (13.6), 1.658 (13.7), 1.625 (13.6), 1.892 (13.6), 1.802 (13.5), 2.090 (13.4), 2.883 (13.4), 2.444 (13.5), 3.057 (13.1), 4.783 (12.6). Thus the land price per square meter increases by $9.8 \%$ for a 4 story building over a 3 story building, increases by $16.1 \%$ for a 5 story building over a 3 story building and so on. The rate of increase is almost monotonic.
} 
the first method of imputation which set the share of total land to unit $\mathrm{n}$ in period $\mathrm{t}, \mathrm{L}_{\mathrm{Stn}}$, equal to $\left(\mathrm{S}_{\mathrm{tn}} / \mathrm{TS}_{\mathrm{tn}}\right) \mathrm{TL}_{\mathrm{tn}}$ whereas the second method set $\mathrm{L}_{\mathrm{Ntn}}$ equal to $\left(1 / \mathrm{N}_{\mathrm{tn}}\right) \mathrm{TL}_{\mathrm{tn}}$. In this section, we set the land imputation for unit $\mathrm{n}$ in period $\mathrm{t}, \mathrm{L}_{\mathrm{t}}$, equal to a weighted average of the two imputation methods and estimate the best fitting weight, $\lambda$. Thus we define:

(16) $\mathrm{L}_{\mathrm{tn}}(\lambda)=\left[\lambda\left(\mathrm{S}_{\mathrm{tn}} / \mathrm{TS}_{\mathrm{tn}}\right)+(1-\lambda)\left(1 / \mathrm{N}_{\mathrm{tn}}\right)\right] \mathrm{TL}_{\mathrm{tn}}$; $\mathrm{t}=1, \ldots, 61 ; \mathrm{n}=1, \ldots, \mathrm{N}(\mathrm{t})$.

The new nonlinear regression model is the following one:

$$
\begin{aligned}
\mathrm{V}_{\mathrm{Ltn}}=\alpha_{\mathrm{t}}\left(\sum_{\mathrm{j}=1}{ }^{9} \omega_{\mathrm{j}} \mathrm{D}_{\mathrm{W}, \mathrm{tn}, \mathrm{j}}\right)\left(\sum_{\mathrm{h}=1}{ }^{14} \chi_{\mathrm{h}} \mathrm{D}_{\mathrm{TH}, \mathrm{tn}, \mathrm{h}}\right)\left(1+\gamma\left(\mathrm{H}_{\mathrm{tn}}-3\right)\right) \mathrm{L}_{\mathrm{tn}}(\lambda)+\varepsilon_{\mathrm{tn}} ; \\
\mathrm{t}=1, \ldots, 61 ; \mathrm{n}=1, \ldots, \mathrm{N}(\mathrm{t})
\end{aligned}
$$

where $\mathrm{L}_{\mathrm{tn}}(\lambda)$ is defined by (16). Again, the normalizations (14) on the parameters in (17) were imposed.

The $\mathrm{R}^{2}$ for this model turned out to be 0.3021 and the $\log$ likelihood was -24644.8 , a huge increase of 161.0 over the LL of the model defined by (15) for the addition of one new parameter. The estimated $\lambda$ turned out to be $\lambda^{*}=0.3636(t=9.84)$ so that the very simple land imputation method that just divided the total land plot size by the number of units in the building got a higher weight (0.6364) than the weight for the floor space allocation method (0.3636).

\section{The Introduction of the Number of Units in the Building as an Explanatory Variable}

Conditional on the land area of the building, we would expect the sold unit's land imputation value to increase as the number of units in the building increases. Thus in this section, we introduce the total number of units in the building, $\mathrm{N}_{\mathrm{tn}}$, as a quality adjustment variable for the imputed land value of a condo unit. We will introduce this variable as a continuous variable in the same manner that we introduced the unit's height, $\mathrm{H}_{\mathrm{tn}}$, as a continuous variable in the nonlinear regression model. The range of the number of units in the building in our sample was from 11 to 154 . Thus we will introduce the term $1+\kappa\left(\mathrm{N}_{\mathrm{tn}}-11\right)$ as an explanatory term in the nonlinear regression. The new parameter $\kappa$ is the percentage increase in the unit's imputed value of land as the number of units in the building grows by one unit.

The new nonlinear regression model is the following one:

$$
\begin{array}{r}
\mathrm{V}_{\mathrm{Ltn}}=\alpha_{\mathrm{t}}\left(\sum_{\mathrm{j}=1}^{9} \omega_{\mathrm{j}} \mathrm{D}_{\mathrm{W}, \mathrm{tn}, \mathrm{j}}\right)\left(\sum_{\mathrm{h}=1}{ }^{14} \chi_{\mathrm{h}} \mathrm{D}_{\mathrm{TH}, \mathrm{tn}, \mathrm{h}}\right)\left(1+\gamma\left(\mathrm{H}_{\mathrm{tn}}-3\right)\right)\left(1+\kappa\left(\mathrm{N}_{\mathrm{tn}}-11\right)\right) \mathrm{L}_{\mathrm{tn}}(\lambda)+\varepsilon_{\mathrm{tn}} \\
\mathrm{t}=1, \ldots, 61 ; \mathrm{n}=1, \ldots, \mathrm{N}(\mathrm{t})
\end{array}
$$

where $\mathrm{L}_{\mathrm{tn}}(\lambda)$ is defined by (16). Again, the normalizations (14) on the parameters in (18) were imposed.

The $\mathrm{R}^{2}$ for this model turned out to be 0.3081 and the $\log$ likelihood was -24604.4 , a substantial increase of 40.4 over the LL of the model defined by (17) for the addition of 
one new parameter. The estimated number of units parameter turned out to be $\kappa^{*}=$ $-0.00183(\mathrm{t}=-10.65)$, a rather small negative number (which did not align with our prior beliefs that this parameter would be positive). ${ }^{22}$

\section{The Introduction of Excess Land as an Explanatory Variable}

The footprint of a building is the area of the land that directly supports the structure. An approximation to the footprint land for unit $\mathrm{n}$ in period $\mathrm{t}$ is the total structure area $\mathrm{TS}_{\mathrm{tn}}$ divided by the total number of stories in the structure $\mathrm{TH}_{\mathrm{tn}}$. If we subtract footprint land from the total land area, $\mathrm{TL}_{\mathrm{tn}}$, we get excess land ${ }^{23} \mathrm{EL}_{\mathrm{tn}}$ defined as follows:

(19) $\mathrm{EL}_{\mathrm{tn}} \equiv \mathrm{TL}_{\mathrm{tn}}-\left(\mathrm{TS}_{\mathrm{tn}} / \mathrm{TH}_{\mathrm{tn}}\right)$;

$\mathrm{t}=1, \ldots, 61 ; \mathrm{n}=1, \ldots, \mathrm{N}(\mathrm{t})$.

In our sample, excess land ranged from $47.26 \mathrm{~m}^{2}$ to $2912.6 \mathrm{~m}^{2}$. We grouped our observations into 10 categories, depending on the amount of excess land that pertained to each observation. Group 1 consists of observations tn where $\mathrm{EL}_{\mathrm{tn}}<200 ; 2$ : observations such that $200 \leq \mathrm{EL}_{\mathrm{tn}}<400 ; 3: 400 \leq \mathrm{EL}_{\mathrm{tn}}<600 ; 4: 600 \leq \mathrm{EL}_{\mathrm{tn}}<800 ; 5: 800 \leq \mathrm{EL}_{\mathrm{tn}}<$ 1000; 6: $1000 \leq \mathrm{EL}_{\mathrm{tn}}<1200 ; 7: 1200 \leq \mathrm{EL}_{\mathrm{tn}}<1500 ; 8: 1500 \leq \mathrm{EL}_{\mathrm{tn}}<2000 ; 9: 2000 \leq$ $\mathrm{EL}_{\mathrm{tn}}<2500$ and Group 10: $2500 \leq \mathrm{EL}_{\mathrm{tn}}$. Now define the excess land dummy variables, $D_{\text {EL,tn, }}$, as follows: $\mathrm{t}=1, \ldots, 61 ; \mathrm{n}=1, \ldots, \mathrm{N}(\mathrm{t}) ; \mathrm{m}=1, \ldots, 10$ :

(20) $\mathrm{D}_{\mathrm{EL}, \mathrm{tn}, \mathrm{m}} \equiv 1$ if observation $\mathrm{n}$ in period $\mathrm{t}$ is in excess land group $\mathrm{m}$;

$\equiv 0$ if observation $\mathrm{n}$ in period $\mathrm{t}$ is $n o t$ in excess land group $\mathrm{m}$.

We will use the above dummy variables as adjustment factors to the price of land. A priori, we expected that an increase in the amount of excess land (holding constant other factors) would lead to an increase in the overall price of land per $\mathrm{m}^{2}$ since more excess land should lead to better views and possibly more amenities for each condo unit and thus increase the price of land. In fact, the opposite happened; the more excess land a property possessed, the lower was the per meter squared value of land for that property.

The new excess land regression model is the following one:

(21) $\mathrm{V}_{\mathrm{Ltn}}=\alpha_{\mathrm{t}}\left(\sum_{\mathrm{j}=1}{ }^{9} \omega_{\mathrm{j}} \mathrm{D}_{\mathrm{W}, \mathrm{tn}, \mathrm{j}}\right)\left(\sum_{\mathrm{h}=1}{ }^{14} \chi_{\mathrm{h}} \mathrm{D}_{\mathrm{TH}, \mathrm{tn}, \mathrm{h}}\right)\left(\sum_{\mathrm{m}=1}{ }^{10} \mu_{\mathrm{m}} \mathrm{D}_{\mathrm{EL}, \mathrm{tn}, \mathrm{m}}\right) \times$ $\left(1+\gamma\left(\mathrm{H}_{\mathrm{tn}}-3\right)\right)\left(1+\kappa\left(\mathrm{N}_{\mathrm{tn}}-11\right)\right) \mathrm{L}_{\mathrm{tn}}(\lambda)+\varepsilon_{\mathrm{tn}} ; \quad \mathrm{t}=1, \ldots, 61 ; \mathrm{n}=1, \ldots, \mathrm{N}(\mathrm{t})$

where $\mathrm{L}_{\mathrm{tn}}(\lambda)$ is defined by (16). Not all of the parameters in (21) can be identified so we impose the following normalizations on the parameters in (21):

(22) $\alpha_{1} \equiv 1 ; \chi_{1} \equiv 1 ; \mu_{1} \equiv 1$.

The $\mathrm{R}^{2}$ for this model turned out to be 0.5259 and the $\log$ likelihood was -23584.06 , a huge increase of 1020.3 over the LL of the model defined by (17) for the addition of 9

\footnotetext{
${ }^{22}$ However, in subsequent models, this parameter did become positive and significant.

${ }^{23}$ This is land that is usable for purposes other than the direct support of the structure on the land plot.
} 
new parameters. The number of units parameter now turns out to be $\kappa^{*}=0.0205(\mathrm{t}=$ 18.45 ) so as the number of units in the structure grows by one, the land value grows by $2.05 \%$. A full listing of the estimated parameters for this model may be found in the Table below.

Table 2: Estimated Coefficients for the Hedonic Regression Model Defined by (21)

\begin{tabular}{|c|c|c|c|c|c|c|c|c|}
\hline Coef & Estimate & t stat & Coef & Estimate & t stat & Coef & Estimate & t stat \\
\hline$\omega_{1}{ }^{*}$ & $\mathbf{5 7 . 6 9 7}$ & 15.26 & $\alpha_{25}{ }^{*}$ & 0.8621 & 18.93 & $\alpha_{57}{ }^{*}$ & 0.9310 & 22.42 \\
\hline$\omega_{2}{ }^{*}$ & 53.448 & 15.48 & $\alpha_{26}{ }^{*}$ & 0.9193 & 19.39 & $\alpha_{58}{ }^{*}$ & 0.9670 & 23.35 \\
\hline$\omega_{3}{ }^{*}$ & 57.379 & 15.10 & $\alpha_{27}{ }^{*}$ & 0.8492 & 16.81 & $\alpha_{59}{ }^{*}$ & 1.0016 & 22.27 \\
\hline$\omega_{4}{ }^{*}$ & 47.740 & 15.08 & $\alpha_{28}{ }^{*}$ & 0.9380 & 18.08 & $\alpha_{60}{ }^{*}$ & 1.0787 & 23.51 \\
\hline$\omega_{5}{ }^{*}$ & $\begin{array}{l}51.530 \\
\end{array}$ & 15.57 & $\alpha_{29}{ }^{*}$ & 1.0095 & 21.02 & $\alpha_{61}{ }^{*}$ & 1.0473 & 23.90 \\
\hline$\omega_{6}{ }^{*}$ & 62.170 & 15.82 & $\alpha_{30}{ }^{*}$ & 1.0632 & 20.28 & $\chi_{2}{ }^{*}$ & 1.1441 & 16.32 \\
\hline$\omega_{7}{ }^{*}$ & 32.959 & 15.29 & $\alpha_{31}{ }^{*}$ & 1.0981 & 19.49 & $\chi_{3}{ }^{*}$ & 1.2530 & 18.42 \\
\hline$\omega_{8}{ }^{*}$ & 39.003 & 15.30 & $\alpha_{32}{ }^{*}$ & 1.0753 & 20.59 & $\chi_{4}{ }^{*}$ & 1.4479 & 18.10 \\
\hline$\omega_{9}{ }^{*}$ & 47.491 & 15.43 & $\alpha_{33}{ }^{*}$ & 1.1116 & 21.18 & $\chi_{5}{ }^{*}$ & 1.6243 & 18.59 \\
\hline $\boldsymbol{\alpha}_{2}{ }^{*}$ & 0.9875 & 18.39 & $\alpha_{34}{ }^{*}$ & 1.1750 & 22.71 & $\chi_{6}{ }^{*}$ & 1.7804 & 18.64 \\
\hline$\alpha_{3}{ }^{*}$ & 0.8782 & 14.20 & $\alpha_{35}{ }^{*}$ & 1.0734 & 20.83 & $\chi_{7}{ }^{*}$ & 1.8723 & 18.09 \\
\hline$\alpha_{4}{ }^{*}$ & 0.9894 & 19.31 & $\alpha_{36}{ }^{*}$ & 0.9575 & 20.16 & $\chi_{8}{ }^{*}$ & 9440 & 18.21 \\
\hline$\alpha_{5}{ }^{*}$ & 1.0279 & 20.08 & $\alpha_{37^{*}}$ & 0.7893 & 16.00 & $\chi_{9}{ }^{*}$ & 1.8659 & 17.70 \\
\hline$\alpha_{6}{ }^{*}$ & 0.9397 & 17.03 & $\alpha_{38}{ }^{*}$ & $\begin{array}{l}0.8538 \\
\end{array}$ & 17.67 & $\chi_{10}{ }^{*}$ & 2.0959 & 17.80 \\
\hline $\boldsymbol{\alpha}_{7}{ }^{*}$ & 0.8247 & 15.46 & $\alpha_{39}{ }^{*}$ & 0.8797 & 18.25 & $\chi_{11}{ }^{*}$ & 2.3800 & 17.41 \\
\hline$\alpha_{8}{ }^{*}$ & 0.8204 & 16.91 & $\alpha_{40}{ }^{*}$ & 0.9223 & 17.90 & $\chi_{12}{ }^{*}$ & 2.2922 & 17.55 \\
\hline$\alpha_{9}{ }^{*}$ & 0.9332 & 18.94 & $\alpha_{41}{ }^{*}$ & 0.9991 & 18.26 & $\chi_{13}{ }^{*}$ & 2.5254 & 17.04 \\
\hline$\alpha_{10}{ }^{*}$ & $\mathbf{0 . 8 0 3 3}$ & 16.17 & $\alpha_{42}{ }^{*}$ & 1.0946 & 20.18 & $\chi_{14}^{*}$ & 3.0692 & 14.04 \\
\hline$\alpha_{11}{ }^{*}$ & 0.9192 & 16.79 & $\alpha_{43}{ }^{*}$ & 1.0883 & 20.37 & $\gamma^{*}$ & 0.0166 & 7.16 \\
\hline$\alpha_{12}{ }^{*}$ & 0.8466 & 15.08 & $\alpha_{44}{ }^{*}$ & 1.0944 & 19.57 & $\kappa^{*}$ & 0.0205 & 18.45 \\
\hline$\alpha_{13}{ }^{*}$ & 0.9045 & 16.70 & $\alpha_{45}{ }^{*}$ & 1.1586 & 19.38 & $\mu_{2}{ }^{*}$ & 0.6802 & 46.89 \\
\hline$\alpha_{14}{ }^{*}$ & 0.8670 & 15.00 & $\alpha_{46}{ }^{*}$ & 1.1636 & 20.33 & $\mu_{3}{ }^{*}$ & 0.5372 & 45.49 \\
\hline$\alpha_{15}{ }^{*}$ & 0.8045 & 12.96 & $\alpha_{47^{*}}$ & 1.0981 & 19.87 & $\mu_{4}{ }^{*}$ & 0.4382 & 41.06 \\
\hline$\alpha_{16}{ }^{*}$ & 0.8490 & 15.82 & $\alpha_{48}{ }^{*}$ & 1.0545 & 22.50 & $\mu_{5}{ }^{*}$ & 0.3824 & 37.94 \\
\hline$\alpha_{17}^{*}$ & 0.8498 & 16.42 & $\alpha_{49}{ }^{*}$ & 0.9837 & 22.43 & $\mu_{6}{ }^{*}$ & 0.3510 & 35.22 \\
\hline$\alpha_{18}{ }^{*}$ & 0.7550 & $\begin{array}{ll}13.88 \\
\end{array}$ & $\alpha_{50}{ }^{*}$ & 1.0026 & 22.47 & $\mu_{7}{ }^{*}$ & $\begin{array}{l}0.2918 \\
\end{array}$ & 33.61 \\
\hline$\alpha_{19}{ }^{*}$ & 0.8271 & 14.25 & $\alpha_{51}{ }^{*}$ & 0.9491 & 22.15 & $\mu_{8}{ }^{*}$ & 0.2491 & 32.39 \\
\hline$\alpha_{20}{ }^{*}$ & 0.8129 & 17.23 & $\alpha_{52}{ }^{*}$ & 1.0363 & 23.94 & $\mu_{9}{ }^{*}$ & 0.2067 & 28.25 \\
\hline$\alpha_{21}{ }^{*}$ & 0.7998 & 17.15 & $\alpha_{53}{ }^{*}$ & 0.9936 & 22.29 & $\mu_{10}{ }^{*}$ & 0.1611 & 21.71 \\
\hline$\alpha_{22}{ }^{*}$ & 0.8528 & 15.70 & $\alpha_{54}{ }^{*}$ & 0.9667 & 23.47 & $\lambda^{*}$ & 0.5430 & 18.76 \\
\hline$\alpha_{23}{ }^{*}$ & 0.9223 & 19.83 & $\alpha_{55}{ }^{*}$ & & 22.26 & & & \\
\hline$\alpha_{24}{ }^{*}$ & 0.9193 & 19.39 & $\alpha_{56}{ }^{*}$ & 0.9499 & 23.38 & & & \\
\hline
\end{tabular}

The $\omega_{j}{ }^{*}$ are the parameters that correspond to the Ward dummy variables; $\alpha_{t}{ }^{*}$ is the constant quality average land price for the condo units that sold in quarter $\mathrm{t}$; the $\chi_{\mathrm{h}}{ }^{*}$ are the land price premiums that accrue to increases in the building height; the $\mu_{\mathrm{m}}{ }^{*}$ are the land price discounts that are associated with increases in excess land; $\lambda^{*}$ is the weight for the structure area imputation method $\left(\lambda^{*}\right.$ is now 0.5430$) ; \gamma^{*}$ (equal to $1.66 \%$ ) is the rate of land price increase as the height of the unit increases by one story and $\kappa^{*}$ (equal to $2.05 \%$ ) is the rate of land price increase as the number of units in the building increases by one. Note that the excess land coefficients $\mu_{\mathrm{m}}{ }^{*}$ steadily decrease as the amount of excess land increases.

The jump in $\log$ likelihood and $\mathrm{R}^{2}$ due to the addition of the excess land dummy variables is rather remarkable. What this model seems to show is that increases in excess land (land 
which is not used to directly support the structure) are not valued by purchasers of Japanese condo units. However, this interpretation is not quite true. Think of two properties in the same neighbourhood which have exactly the same structure on the land plot. Property A has no excess land while property B has a lot of excess land. The excess land on property B will have some value but this value per square meter will be less than the value of land per square meter for property $A$. Thus the average value of land per square meter on property B will be less than that of property A.

\section{The Introduction of Subway Travel Times and Facing South as Explanatory Variables}

There are three additional explanatory variables in our data set that may affect the price of land. Recall that TW was defined as walking time in minutes to the nearest subway station; TT as the subway running time in minutes to the Tokyo station from the nearest station and the SOUTH dummy variable is equal to 1 if the unit faces south and 0 otherwise. Let $\mathrm{D}_{\mathrm{S}, \mathrm{tn}, 2}$ equal the SOUTH dummy variable for sale $\mathrm{n}$ in quarter $\mathrm{t}$. Define $\mathrm{D}_{\mathrm{S}, \mathrm{n}, 2}=1-\mathrm{D}_{\mathrm{S}, \mathrm{tn}, 1}$. TW ranges from 1 to 19 minutes while TT ranges from 12 to 48 minutes. These new variables are inserted into the nonlinear regression model (21) in the following manner:

$$
\begin{aligned}
& \mathrm{V}_{\mathrm{Ltn}}= \alpha_{\mathrm{t}}\left(\sum_{\mathrm{j}=1}{ }^{9} \omega_{\mathrm{j}} \mathrm{D}_{\mathrm{W}, \mathrm{tn}, \mathrm{j}}\right)\left(\sum_{\mathrm{h}=1}^{14} \chi_{\mathrm{h}} \mathrm{D}_{\mathrm{TH}, \mathrm{tn}, \mathrm{h}}\right)\left(\sum_{\mathrm{m}=1}{ }^{10} \mu_{\mathrm{m}} \mathrm{D}_{\mathrm{EL}, \mathrm{tn}, \mathrm{m}}\right)\left(\phi_{\mathrm{l}} \mathrm{D}_{\mathrm{S}, \mathrm{tn}, 1}+\phi_{2} \mathrm{D}_{\mathrm{S}, \mathrm{tn}, 2}\right) \\
& \times\left(1+\gamma\left(\mathrm{H}_{\mathrm{tn}}-3\right)\right)\left(1+\kappa\left(\mathrm{N}_{\mathrm{tn}}-11\right)\right)\left(1+\eta\left(\mathrm{TW}_{\mathrm{tn}}-1\right)\right)\left(1+\theta\left(\mathrm{TT}_{\mathrm{tn}}-12\right)\right) \mathrm{L}_{\mathrm{tn}}(\lambda)+\varepsilon_{\mathrm{tn}} ; \\
& \mathrm{t}=1, \ldots, 61 ; \mathrm{n}=1, \ldots, \mathrm{N}(\mathrm{t})
\end{aligned}
$$

where $\mathrm{L}_{\mathrm{tn}}$ is defined by (16). Not all of the parameters in (23) can be identified so we impose the normalizations (24) on the parameters in (23):

(24) $\alpha_{1} \equiv 1 ; \chi_{1} \equiv 1 ; \mu_{1} \equiv 1 ; \phi_{1} \equiv 1$

The $\mathrm{R}^{2}$ for this model turned out to be 0.6308 and the log likelihood was -23178.30 , a huge increase of 405.8 over the LL of the model defined by (21) for the addition of 3 new parameters. The estimated facing south parameter is $\phi_{2}{ }^{*}=1.0294(\mathrm{t}=120.6)$ so the land value of a condo unit that faces south increases by $2.94 \%$. The walking to the subway parameter turns out to be $\eta^{*}=-0.0176(t=-26.7)$ so that an extra minute of walking time reduces the land value component of the condo by $1.76 \%$. The travel time to the Tokyo Central Station parameter is $\theta^{*}=-0.0128(\mathrm{t}=-27.4)$ so that an extra minute of travel time reduces the land value component of the condo by $1.28 \%$. It can be seen that these three additional explanatory variables have explanatory power!

In the following sections, we switch from imputed land value $V_{L t n}$ as the dependent variable in the regressions to the selling price of the property, $\mathrm{V}_{\mathrm{tn}}$. We use the estimated values for the coefficients in (23) as starting values in the nonlinear regression which follows.

\section{Using the Selling Price as the Dependent Variable}


Our new model in this section uses $V_{\text {tn }}$ as the dependent variable and uses the same specification for the land component of the property that we used in the previous section but now we add the term $(1.1) \mathrm{p}_{\mathrm{St}}(1-\delta)^{\mathrm{A}(\mathrm{t}, \mathrm{n})} \mathrm{S}_{\mathrm{tn}}$ to account for the structure component of the value of the condo unit. Note that we will now estimate the annual depreciation rate $\delta$ in our new model, rather than assuming that it was equal to 3\%. Thus the number of unknown parameters in our new model increased from 97 to 98.

The $\mathrm{R}^{2}$ for this new model turned out to be 0.8190 and the log likelihood was -23164.33 . This LL cannot be compared with the LL in the previous model, because the dependent variable has changed. The estimated depreciation rate was $\delta^{*}=0.0367(\mathrm{t}=27.1)$. This estimated annual depreciation rate of $3.67 \%$ is higher than our earlier assumed rate of $3.00 \%$. Note that the $\mathrm{R}^{2}$ is now satisfactory; i.e., our model is explaining a substantial fraction of the variation in condo prices.

\section{The Introduction of Explanatory Variables to Adjust the Quality of the Structure}

In this section, we introduce the number of bedrooms variable, $\mathrm{NB}_{\mathrm{tn}}$, and the reinforced concrete construction $\mathrm{SCR}_{\mathrm{nt}}$ dummy variable as quality adjusters for the value of the structure. Recall that $\mathrm{SCR}_{\mathrm{nt}}=1$ if the building for condo sale $\mathrm{n}$ in quarter $\mathrm{t}$ used reinforced concrete construction. Recall also that $\mathrm{NB}_{\mathrm{nt}}$ is the number of bedrooms for condo $\mathrm{n}$ sold in quarter $\mathrm{t}$ and this variable ranged from 2 to 5 . We grouped our observations into 3 categories. Group 1 consists of observations tn where $\mathrm{NB}_{\mathrm{nt}}=2$, Group 2 consists of observations tn where $\mathrm{NB}_{\mathrm{nt}}=3$ and Group 3 consists of observations tn where $\mathrm{NB}_{\mathrm{nt}}=4$ or $5 .{ }^{24}$ Now define the bedroom dummy variables, $\mathrm{D}_{\mathrm{B}, \mathrm{tn}, \mathrm{i}}$, as follows: $\mathrm{t}$ $=1, \ldots, 61 ; \mathrm{n}=1, \ldots, \mathrm{N}(\mathrm{t}) ; \mathrm{i}=1,2,3$ :

(25) $\mathrm{D}_{\mathrm{B}, \mathrm{tn}, \mathrm{i}} \equiv 1$ if observation $\mathrm{n}$ in period $\mathrm{t}$ is in bedroom Group $\mathrm{i}$; $\equiv 0$ if observation $\mathrm{n}$ in period $\mathrm{t}$ is $n o t$ in bedroom Group $\mathrm{i}$.

We will use the above dummy variables as adjustment factors to the price of the structure. A priori, we expect that an increase in the number of bedrooms would lead to an increase in the value of the structure. Our new hedonic regression model is the following one:

$$
\text { (26) } \begin{aligned}
& \mathrm{V}_{\mathrm{tn}}= \alpha_{\mathrm{t}}\left(\sum_{\mathrm{j}=1}^{9} \omega_{\mathrm{j}} \mathrm{D}_{\mathrm{W}, \mathrm{tn}, \mathrm{j}}\right)\left(\sum_{\mathrm{h}=1}^{14} \chi_{\mathrm{h}} \mathrm{D}_{\mathrm{TH}, \mathrm{tn}, \mathrm{h}}\right)\left(\sum_{\mathrm{m}=1}^{10} \mu_{\mathrm{m}} \mathrm{D}_{\mathrm{EL}, \mathrm{tn}, \mathrm{m}}\right)\left(\phi_{1} \mathrm{D}_{\mathrm{S}, \mathrm{tn}, 1}+\phi_{2} \mathrm{D}_{\mathrm{S}, \mathrm{tn}, 2}\right) \\
& \times\left(1+\gamma\left(\mathrm{H}_{\mathrm{tn}}-3\right)\right)\left(1+\kappa\left(\mathrm{N}_{\mathrm{tn}}-11\right)\right)\left(1+\eta\left(\mathrm{TW}_{\mathrm{tn}}-1\right)\right)\left(1+\theta\left(\mathrm{TT}_{\mathrm{tn}}-12\right)\right) \mathrm{L}_{\mathrm{tn}}(\lambda) \\
&+(1.1) \mathrm{p}_{\mathrm{St}}(1-\delta)^{\mathrm{A}(\mathrm{t}, \mathrm{n})}\left(1+\sigma \mathrm{OSCR} \mathrm{tn}_{\mathrm{tn}}\right)\left(\Sigma_{\mathrm{i}=1}{ }^{3} \rho_{\mathrm{i}} \mathrm{D}_{\mathrm{B}, \mathrm{tn}, \mathrm{i}}\right) \mathrm{S}_{\mathrm{tn}}+\varepsilon_{\mathrm{tn}} ; \\
& \mathrm{t}=1, \ldots, 61 ; \mathrm{n}=1, \ldots, \mathrm{N}(\mathrm{t})
\end{aligned}
$$

with the following normalizations on the parameters in (26):

\footnotetext{
${ }^{24}$ Initially, we had separate Groups for 4 and 5 bedroom condominiums but we found that the estimated parameters associated with the corresponding dummy variables were almost equal (the estimated coefficients were equal to 1.254 and 1.233 with t statistics equal to 6.1 and 6.0). When we combined 4 and 5 bedroom apartments into a single Group, the $\mathrm{R}^{2}$ and $\log$ likelihood for the resulting model (26) remained the same as the more general model with 4 bedroom groups.
} 
$\alpha_{1} \equiv 1 ; \chi_{1} \equiv 1 ; \mu_{1} \equiv 1 ; \phi_{1} \equiv 1 ; \rho_{1} \equiv 1$

The $\mathrm{R}^{2}$ for this new model turned out to be 0.8298 and the log likelihood was -23039.88 , an increase in log likelihood of 124.4 for the addition of 3 new parameters. The estimated parameter for having reinforced concrete construction was $\sigma^{*}=0.0345(\mathrm{t}=2.52)$ so that the structure value of the sold condo unit increases by $3.45 \%$ if the building used reinforced (with steel bars) concrete construction. The estimated number of bedroom parameters turned out to be $\rho_{2}{ }^{*}=1.143(\mathrm{t}=107.0)$ and $\rho_{3}{ }^{*}=1.254(\mathrm{t}=61.3)$. Thus as we move from a 2 bedroom condo to a 3 bedroom condo, the value of the structure increases by $14.3 \%$. As we move from a 2 bedroom condo to a 4 or 5 bedroom condo, the value of the structure increases by $25.4 \%$. Thus the new parameters have reasonable values and add to the explanatory value of the regression. A complete listing of the estimated coefficients and their standard errors can be found in Table $3 .^{25}$ It can be seen that all of the estimated coefficients are reasonable and are not too different from the corresponding coefficients that were estimated in the previous models.

We estimated a generalization of the model defined by (25) by replacing the condo floor space area variable $S_{\mathrm{tn}}$ by $\beta \mathrm{S}_{\mathrm{tn}}$ where $\beta$ is the general quality adjustment parameter which appeared in equations (4) and (5) in section 3 above. The resulting model (with one extra parameter) led to an $\mathrm{R}^{2}$ of 0.8507 and a log likelihood of -22822.99 , an increase of 216.9 for the addition of one new parameter. Unfortunately, the resulting estimate for the new parameter was $\beta^{*}=1.6240(\mathrm{t}=72.1)$, which is too high (it should be around unity). This high estimate led to a property structure values which were too high and to land values which were too low. ${ }^{26}$ The high estimate also increased the volatility of land prices to an unreasonable degree. Thus we prefer the present model (25) which led to much more reasonable land prices.

Table 3: Estimated Coefficients for the Regression Model Defined by (26)

\begin{tabular}{|c|c|c|c|c|c|c|c|c|}
\hline Coef & Estimate & t stat & Coef & Estimate & t stat & Coef & Estimate & t stat \\
\hline$\omega_{1}{ }^{*}$ & 67.228 & 17.32 & $\alpha_{27}{ }^{*}$ & 0.8752 & 20.93 & $\alpha_{61}{ }^{*}$ & 1.1090 & 29.70 \\
\hline$\omega_{2}{ }^{*}$ & 66.127 & 17.34 & $\alpha_{28}{ }^{*}$ & 0.9652 & 22.84 & $\chi_{2}{ }^{*}$ & 1.2762 & 20.60 \\
\hline$\omega_{3}{ }^{*}$ & 68.716 & 17.09 & $\alpha_{29}{ }^{*}$ & 1.0077 & 26.09 & $\chi_{3}{ }^{*}$ & 1.3615 & 23.34 \\
\hline$\omega_{4}{ }^{*}$ & 58.664 & 16.99 & $\alpha_{30}{ }^{*}$ & 1.0574 & 25.76 & $\chi_{4}{ }^{*}$ & 1.6139 & 23.34 \\
\hline$\omega_{5}{ }^{*}$ & 75.634 & 17.26 & $\alpha_{31}{ }^{*}$ & 1.1213 & 24.71 & $\chi_{5}{ }^{*}$ & 1.7287 & 23.56 \\
\hline$\omega_{6}{ }^{*}$ & 100.970 & 17.17 & $\alpha_{32}{ }^{*}$ & 1.0851 & 26.22 & $\chi_{6}{ }^{*}$ & 1.8484 & 23.36 \\
\hline$\omega_{7}{ }^{*}$ & 44.897 & 16.63 & $\alpha_{33}{ }^{*}$ & 1.1582 & 27.04 & $\chi_{7}{ }^{*}$ & 1.9079 & 22.93 \\
\hline$\omega_{8}{ }^{*}$ & 51.008 & 16.96 & $\alpha_{34}{ }^{*}$ & 1.1906 & 28.74 & $\chi_{8}{ }^{*}$ & 1.9669 & 22.79 \\
\hline$\omega_{9}{ }^{*}$ & 62.921 & 17.19 & $\alpha_{35}{ }^{*}$ & 1.0547 & 26.13 & $\chi_{9}{ }^{*}$ & 1.9250 & 22.37 \\
\hline$\alpha_{2}{ }^{*}$ & 0.9703 & 23.24 & $\alpha_{36}{ }^{*}$ & 0.9525 & 25.38 & $\chi_{10}{ }^{*}$ & 2.0383 & 22.01 \\
\hline$\alpha_{3}{ }^{*}$ & 0.8540 & 18.31 & $\alpha_{37}{ }^{*}$ & 0.8510 & 19.80 & $\chi_{11}{ }^{*}$ & 2.2529 & 21.65 \\
\hline$\alpha_{4}{ }^{*}$ & 0.9707 & 24.27 & $\alpha_{38}{ }^{*}$ & 0.8568 & 22.59 & $\chi_{12}{ }^{*}$ & 2.1819 & 21.75 \\
\hline$\alpha_{5}{ }^{*}$ & 0.9993 & 25.75 & $\alpha_{39}{ }^{*}$ & 0.8922 & 22.77 & $\chi_{13}{ }^{*}$ & 2.3523 & 20.67 \\
\hline$\alpha_{6}{ }^{*}$ & 0.9657 & 21.77 & $\alpha_{40}{ }^{*}$ & 0.9416 & 22.34 & $\chi_{14}{ }^{*}$ & 2.9889 & 17.22 \\
\hline$\alpha_{7}{ }^{*}$ & 0.8206 & 20.29 & $\alpha_{41}{ }^{*}$ & 1.0059 & 23.36 & $\gamma^{*}$ & 0.0158 & 8.98 \\
\hline$\alpha_{8}{ }^{*}$ & 0.8451 & 21.87 & $\alpha_{42}{ }^{*}$ & 1.0844 & 25.51 & $\kappa^{*}$ & 0.0165 & 19.18 \\
\hline
\end{tabular}

\footnotetext{
${ }^{25}$ The standard errors are equal to the estimated coefficients divided by the corresponding listed $t$ statistics.

${ }^{26} \mathrm{We}$ consulted with Tokyo real estate experts to determine reasonable land and structure shares of property value for apartment buildings in Tokyo. Our present model generates shares that fall into the reasonable range. These shares are listed in Table A2 in the Appendix.
} 


\begin{tabular}{|c|c|c|c|c|c|c|c|c|}
\hline$\alpha_{9}{ }^{*}$ & 0.9315 & 23.84 & $\alpha_{43}{ }^{*}$ & 1.1276 & 25.96 & $\mu_{2}{ }^{*}$ & 0.7429 & 56.73 \\
\hline$\alpha_{10}{ }^{*}$ & 0.7932 & 20.41 & $\alpha_{44}{ }^{*}$ & 1.2079 & 24.74 & $\mu_{3}{ }^{*}$ & 0.5962 & 52.81 \\
\hline$\alpha_{11}{ }^{*}$ & $\mathbf{0 . 8 8 9 7}$ & 19.71 & $\alpha_{45}{ }^{*}$ & 1.2352 & 24.83 & $\mu_{4}{ }^{*}$ & 0.4987 & 47.63 \\
\hline$\alpha_{12}{ }^{*}$ & 0.8540 & 18.65 & $\alpha_{46}{ }^{*}$ & 1.2378 & 25.97 & $\mu_{5}{ }^{*}$ & 0.4468 & 43.10 \\
\hline$\alpha_{13}{ }^{*}$ & 0.9090 & 21.34 & $\alpha_{47}{ }^{*}$ & 1.1377 & 25.46 & $\mu_{6}{ }^{*}$ & 0.4086 & 38.60 \\
\hline$\alpha_{14}{ }^{*}$ & 0.8561 & 19.12 & $\alpha_{48}{ }^{*}$ & 1.1335 & 28.05 & $\mu_{7}{ }^{*}$ & 0.3475 & 38.17 \\
\hline$\alpha_{15}{ }^{*}$ & $\begin{array}{l}0.8302 \\
\end{array}$ & 16.79 & $\alpha_{49}{ }^{*}$ & 1.0488 & 27.92 & $\mu_{8}{ }^{*}$ & 0.2983 & 35.46 \\
\hline$\alpha_{16}{ }^{*}$ & 0.8275 & 19.87 & $\alpha_{50}{ }^{*}$ & 1.0656 & 28.35 & $\mu_{9}{ }^{*}$ & 0.2497 & 30.36 \\
\hline$\alpha_{17}{ }^{*}$ & 0.8172 & 20.61 & $\alpha_{51}{ }^{*}$ & 0.9841 & 27.67 & $\mu_{10}{ }^{\prime \prime}$ & 0.2163 & 23.80 \\
\hline$\alpha_{18}{ }^{*}$ & 0.7660 & 18.44 & $\alpha_{52}{ }^{*}$ & 1.0600 & 29.64 & $\lambda^{*}$ & 0.4690 & 20.56 \\
\hline$\alpha_{19}{ }^{*}$ & 0.8222 & 17.92 & $\alpha_{53}{ }^{*}$ & 1.0526 & 28.20 & $\phi_{2}{ }^{*}$ & 1.0289 & 132.73 \\
\hline$\alpha_{20}{ }^{*}$ & 0.8102 & 21.46 & $\alpha_{54}{ }^{*}$ & 1.0344 & 29.63 & $\eta^{*}$ & -0.0188 & -29.82 \\
\hline$\alpha_{21}{ }^{*}$ & 0.8234 & 21.68 & $\alpha_{55}{ }^{*}$ & 0.9894 & 27.15 & $\theta^{*}$ & -0.0134 & -30.92 \\
\hline$\alpha_{22}{ }^{*}$ & 0.8193 & 19.32 & $\alpha_{56}{ }^{*}$ & 0.9842 & 28.31 & $\delta^{*}$ & 0.0361 & 28.94 \\
\hline$\alpha_{23}{ }^{*}$ & 0.9182 & 24.80 & $\alpha_{57}{ }^{*}$ & 0.9938 & 27.52 & $\sigma^{*}$ & 0.0345 & 2.52 \\
\hline$\alpha_{24}{ }^{*}$ & 0.9280 & 24.52 & $\alpha_{58}{ }^{*}$ & 1.0273 & 28.33 & $\rho_{2}{ }^{*}$ & 1.1433 & 106.98 \\
\hline$\alpha_{25}{ }^{*}$ & 0.8482 & 23.85 & $\alpha_{59}{ }^{*}$ & 1.0848 & 27.74 & $\rho_{3}{ }^{*}$ & 1.2539 & 61.34 \\
\hline$\alpha_{26}{ }^{*}$ & 0.8647 & 24.07 & $\alpha_{60}{ }^{*}$ & 1.1688 & 28.99 & & & \\
\hline
\end{tabular}

In most countries, movements in property prices are usually quite different in rich neighbourhoods as opposed to poorer neighbourhoods. During booms, property price increases in rich suburbs are usually much bigger and, during busts, property prices fall more in poorer suburbs. Thus in the following section, we will group our nine Wards into rich, medium and poorer Wards according to their estimated ward dummy variables, $\omega_{1}{ }^{*}-$ $\omega_{9}{ }^{*}$ listed in Table 3. Thus Wards 5 and 6 will be classified as rich; 1,2,3,4 and 9 will be classified as medium and Wards 7 and 8 as poorer wards.

\section{Introducing Separate Land Price Parameters for Poor, Medium and Rich Wards.}

Our final model added an additional 120 parameters to the previous model which allowed us to calculate separate land price indexes for poor, medium and rich wards. The terms $\alpha_{\mathrm{t}}\left(\sum_{\mathrm{j}=1}{ }^{9} \omega_{\mathrm{j}} \mathrm{D}_{\mathrm{W}, \mathrm{tn}, \mathrm{j}}\right)$ in (26) were replaced by the following terms:

(28) $\left[\alpha_{\mathrm{tR}}\left(\omega_{5} \mathrm{D}_{\mathrm{W}, \mathrm{tn}, 5}+\omega_{6} \mathrm{D}_{\mathrm{W}, \mathrm{tn}, 6}\right)+\alpha_{\mathrm{tM}}\left(\sum_{\mathrm{j}=1}^{4} \omega_{\mathrm{j}} \mathrm{D}_{\mathrm{W}, \mathrm{tn}, \mathrm{j}}+\omega_{9} \mathrm{D}_{\mathrm{W}, \mathrm{tn}, 9}\right)+\alpha_{\mathrm{tP}}\left(\omega_{7} \mathrm{D}_{\mathrm{W}, \mathrm{tn}, 7}+\omega_{8} \mathrm{D}_{\mathrm{W}, \mathrm{tn}, 8}\right)\right]$.

The normalizations (27) were replaced by the following identifying normalizations:

(29) $\alpha_{1 \mathrm{R}} \equiv 1 ; \alpha_{1 \mathrm{M}} \equiv 1 ; \alpha_{1 \mathrm{P}} \equiv 1 ; \chi_{1} \equiv 1 ; \mu_{1} \equiv 1 ; \phi_{1} \equiv 1 ; \rho_{1} \equiv 1$.

The remaining terms in (26) remained unchanged. There are now 221 parameters in the new model. The $\mathrm{R}^{2}$ for this new model turned out to be 0.8391 and the log likelihood was -22948.76 , an increase of 91.1 for the addition of 120 new parameters. ${ }^{27}$

The sequence of estimated constant quality land prices over the 61 quarters in our sample for rich wards is $1, \alpha_{2 \mathrm{R}}{ }^{*}, \alpha_{3 \mathrm{R}}{ }^{*}, \ldots, \alpha_{61 \mathrm{R}}{ }^{*}$; for medium wards is $1, \alpha_{2 \mathrm{M}}{ }^{*}, \alpha_{3 \mathrm{M}}{ }^{*}, \ldots, \alpha_{61 \mathrm{M}}{ }^{*}$; and for poorer wards is $1, \alpha_{2 \mathrm{P}}{ }^{*}, \alpha_{3 \mathrm{P}}{ }^{*}, \ldots, \alpha_{61 \mathrm{P}}{ }^{*}$. These estimates, along with the estimates for the other parameters in our model, are listed in Table A1 in the Appendix. A chart

\footnotetext{
${ }^{27}$ This is not a very large increase in log likelihood for the addition of so many new parameters. Hence there is a danger that our land price estimates for rich, medium and poor wards may not be reliable.
} 
showing these land price series will be provided in the following section. The Chart indicates that the ward land price indexes that resulted from this model are too variable to be regarded as being reliable. Our conclusion here is that our sample of ward sales is too small to support the estimation of separate ward land price indexes. Hence we regard the estimates from the previous section as being more reliable.

In the following section, we turn our attention to index number issues associated with the models in sections 12 and 13 .

\section{The Construction of Constant Quality Land, Structure and Overall Property Price Indexes}

Recall the model defined by equations (26) in section 12. Once we have estimated the unknown parameters in (26), we can form predicted land and structure values for observation $\mathrm{tn}, \mathrm{V}_{\mathrm{Lnt}}$ and $\mathrm{V}_{\mathrm{Stn}}$, defined as follows. For $\mathrm{t}=1, \ldots, 61 ; \mathrm{n}=1, \ldots, \mathrm{N}(\mathrm{t})$, define: ${ }^{28}$

$$
\begin{aligned}
\mathrm{V}_{\mathrm{Ltn}}= & \alpha_{\mathrm{t}}{ }^{*}\left(\sum_{\mathrm{j}=1}{ }^{9} \omega_{\mathrm{j}}{ }^{*} \mathrm{D}_{\mathrm{W}, \mathrm{tn}, \mathrm{j}}\right)\left(\sum_{\mathrm{h}=1}{ }^{14} \chi_{\mathrm{h}}{ }^{*} \mathrm{D}_{\mathrm{TH}, \mathrm{tn}, \mathrm{h}}\right)\left(\sum_{\mathrm{m}=1}{ }^{10} \mu_{\mathrm{m}}{ }^{*} \mathrm{D}_{\mathrm{EL}, \mathrm{tn}, \mathrm{m}}\right)\left(\phi_{1}{ }^{*} \mathrm{D}_{\mathrm{S}, \mathrm{tn}, 1}+\phi_{2}{ }^{*} \mathrm{D}_{\mathrm{S}, \mathrm{tn}, 2}\right) \\
& \times\left(1+\gamma^{*}\left(\mathrm{H}_{\mathrm{tn}}-3\right)\right)\left(1+\kappa^{*}\left(\mathrm{~N}_{\mathrm{tn}}-11\right)\right)\left(1+\eta^{*}\left(\mathrm{TW}_{\mathrm{tn}}-1\right)\right)\left(1+\theta^{*}\left(\mathrm{TT}_{\mathrm{tn}}-12\right)\right) \mathrm{L}_{\mathrm{tn}}\left(\lambda^{*}\right) \\
\mathrm{V}_{\mathrm{Stn}}= & (1.1) \mathrm{p}_{\mathrm{St}}\left(1-\delta^{*}\right)^{\mathrm{A}(\mathrm{t}, \mathrm{n})}\left(1+\sigma^{*} \mathrm{SCR}_{\mathrm{tn}}\right)\left(\sum_{\mathrm{i}=1}{ }^{3} \rho_{\mathrm{i}}{ }^{*} \mathrm{D}_{\mathrm{B}, \mathrm{tn}, \mathrm{i}}\right) \mathrm{S}_{\mathrm{tn}} .
\end{aligned}
$$

These predicted values for land and structures will be regarded as appropriate imputed values for the land and structure components for each sale. ${ }^{29}$ For each quarter, we can sum up the predicted values for land and structures for each sale in the quarter to form total predicted (or imputed) values for land and structures sold in period $\mathrm{t}, \mathrm{V}_{\mathrm{Lt}}$ and $\mathrm{V}_{\mathrm{St}}$ :

(32) $\mathrm{V}_{\mathrm{Lt}} \equiv \Sigma_{\mathrm{n} \in \mathrm{N}(\mathrm{t})} \mathrm{V}_{\mathrm{Ltn}} ; \mathrm{V}_{\mathrm{St}} \equiv \Sigma_{\mathrm{n} \in \mathrm{N}(\mathrm{t})} \mathrm{V}_{\mathrm{Stn}}$;

$\mathrm{t}=1, \ldots, 61$

Examining equations (30), it can be seen that each term on the right hand side of (30) has $\alpha_{t}{ }^{*}$ as a common factor. Thus we define $\alpha_{t}{ }^{*}$ as the price index for land in period $t, p_{L t}$ and define the corresponding land quantity index for units sold in period $\mathrm{t}, \mathrm{q}_{\mathrm{Lt}}$, as imputed land value for period $t, \mathrm{~V}_{\mathrm{Lt}}$, divided by $\mathrm{p}_{\mathrm{Lt}}$ :

(33) $\mathrm{p}_{\mathrm{Lt}} \equiv \alpha_{\mathrm{t}}^{*} ; \mathrm{q}_{\mathrm{Lt}} \equiv \mathrm{V}_{\mathrm{Lt}} / \mathrm{p}_{\mathrm{Lt}}$;

$\mathrm{t}=1, \ldots, 61$.

The properties sold in any period will be different from the properties sold in any other period. ${ }^{30}$ But the hedonic regression estimated in section 12 can be used to value the land component of all the properties that sold in quarter $t$ (this value is $V_{L t}=\alpha_{t}{ }^{*} q_{L t}$ ) at the prices which prevailed in quarter $\mathrm{s}$, which turns out to be the hypothetical value $\mathrm{V}_{\mathrm{Lst}} \equiv$ $\alpha_{S}{ }^{*} q_{L t}$. Thus a fixed basket (Laspeyres) type price index for land sold in quarter s relative to quarter $\mathrm{t}$ is $\mathrm{P}_{\mathrm{L}} \equiv \mathrm{V}_{\mathrm{Lst}} / \mathrm{V}_{\mathrm{Lt}}=\alpha_{\mathrm{s}}{ }^{*} \mathrm{q}_{\mathrm{Lt}} / \alpha_{\mathrm{t}}{ }^{*} \mathrm{q}_{\mathrm{Lt}}=\alpha_{\mathrm{s}}{ }^{*} / \alpha_{\mathrm{t}}{ }^{*}=\mathrm{p}_{\mathrm{Ls}} / \mathrm{p}_{\mathrm{Lt}}$. This type of price index is

\footnotetext{
${ }^{28}$ We define $\alpha_{1}{ }^{*} \equiv 1, \chi_{1}{ }^{*} \equiv 1, \mu_{1}{ }^{*} \equiv 1, \phi_{1}{ }^{*} \equiv 1$ and $\rho_{1}{ }^{*} \equiv 1$.

${ }^{29}$ The sum of these imputed values differ from the actual property values $V_{\text {tn }}$; i.e., we have $V_{\text {tn }}=V_{\text {Ltn }}+V_{\text {Stn }}$ $+e_{n t}$ where $e_{n t}$ is the observed error term for observation tn in our nonlinear regression.

${ }^{30}$ Even if the same property is sold in two different periods, the properties will in general not be identical due to depreciation and renovations between the two periods.
} 
called a hedonic imputation index. ${ }^{31}$ Similarly, we can look at the properties sold in quarter $\mathrm{s}$ with the total land value of $\mathrm{V}_{\mathrm{Ls}}=\alpha_{\mathrm{S}}{ }^{*} \mathrm{q}_{\mathrm{Ls}}$ and value this group of properties at the land prices of quarter $t$, which turns out to be the hypothetical value $V_{L t s} \equiv \alpha_{t}{ }^{*} q_{L s}$. Thus a fixed basket (Paasche) type price index for land sold in quarter s relative to quarter $\mathrm{t}$ using the land quantities of quarter $s$ is $P_{P} \equiv V_{L s} / V_{L t s}=\alpha_{s}{ }^{*} q_{L s} / \alpha_{t}{ }^{*} q_{L s}=\alpha_{s}{ }^{*} / \alpha_{t}{ }^{*}=p_{L s} / p_{L t}$. This is an alternative hedonic imputation index but it can be seen that it gives rise to the same index as the original hedonic imputation index. The bottom line is that there is a reasonably strong justification for the use of the land price and quantity indexes, $\mathrm{p}_{\mathrm{Lt}}$ and $\mathrm{q}_{\mathrm{Lt}}$, defined by (33) above. $^{32}$

Examining equations (31), it can be seen that each term on the right hand side of (31) has $\mathrm{p}_{\mathrm{St}}$ as a common factor. Thus it is natural to define this common factor $\mathrm{p}_{\mathrm{St}}$ as the price index for structures in period $t$ and define the corresponding structure quantity index for units sold in period $\mathrm{t}, \mathrm{q}_{\mathrm{St}}$, as imputed structure value for period $\mathrm{t}, \mathrm{V}_{\mathrm{St}}$, divided by $\mathrm{p}_{\mathrm{St}}$ :

The land and structure price indexes, $\mathrm{p}_{\mathrm{Lt}}$ and $\mathrm{p}_{\mathrm{St}}$, are plotted on Chart 1 below. ${ }^{33} \mathrm{An}$ overall constant quality property price index $\mathrm{p}_{\mathrm{t}}$ is formed as a chained Fisher index ${ }^{34}$ of the land and structure components ${ }^{35}$ and is also plotted on Chart 1.

Chart 1: Land, Structure and Property Price Indexes $p_{L t}, p_{S t}$ and $p_{t}$ for the Section 12 Model and Land and Property Price Indexes $p_{L t}{ }^{*}$ and $p_{t}{ }^{*}$ for the Section 13 Model

\footnotetext{
${ }^{31}$ This type of index dates back to Court (1939). For more discussion and references to the price index literature, see Chapter 5 in Eurostat (2013).

${ }^{32}$ Basically, the estimated $\alpha_{t}{ }^{*}$ shift the hedonic surface for land in a proportional manner over time and the construction price indexes $\mathrm{p}_{\mathrm{St}}$ shift the hedonic surface for structures in a proportional manner over time. The time dummy hedonic regression approach which uses the log of property price as the dependent variable and introduces time dummy variables into the regression essentially assumes a single proportional shift variable instead of two shift variables (the $\alpha_{t}{ }^{*}$ and the $p_{S t}$ ). Bostic, Longhofer and Redfearn (2007; 185) realized that this was an important limitation of the traditional time dummy model: "In the context of understanding and explaining house price movements, the decomposition of housing into land and improvements is important because it is possible that the value of a parcel of land evolves with a different trajectory than the value of the improvements on it."

${ }^{33}$ These price indexes along with the corresponding quantity indexes are listed in Table A2 of the Appendix.

${ }^{34}$ See Fisher (1922) and Diewert (1992) for the properties of the Fisher ideal index.

${ }^{35}$ The index is set equal to one in period 1 so that $p_{1} \equiv 1$. For quarter $t \geq 2, p_{t}$ is set equal to $p_{t-1}$ times the following link factor: $\left[\left(p_{\mathrm{Lt}} \mathrm{q}_{\mathrm{Lt}}+\mathrm{p}_{\mathrm{St}} \mathrm{q}_{\mathrm{St}}\right)\left(\mathrm{p}_{\mathrm{Lt}} \mathrm{q}_{\mathrm{Lt}-1}+\mathrm{p}_{\mathrm{St}} \mathrm{q}_{\mathrm{St}-1}\right) /\left(\mathrm{p}_{\mathrm{Lt}-1} \mathrm{q}_{\mathrm{Lt}}+\mathrm{p}_{\mathrm{St}-1} \mathrm{q}_{\mathrm{St}}\right)\left(\mathrm{p}_{\mathrm{Lt}-1} \mathrm{q}_{\mathrm{Lt}-1}+\mathrm{p}_{\mathrm{St}-1} \mathrm{q}_{\mathrm{St}-1}\right)\right]^{1 / 2}$.
} 


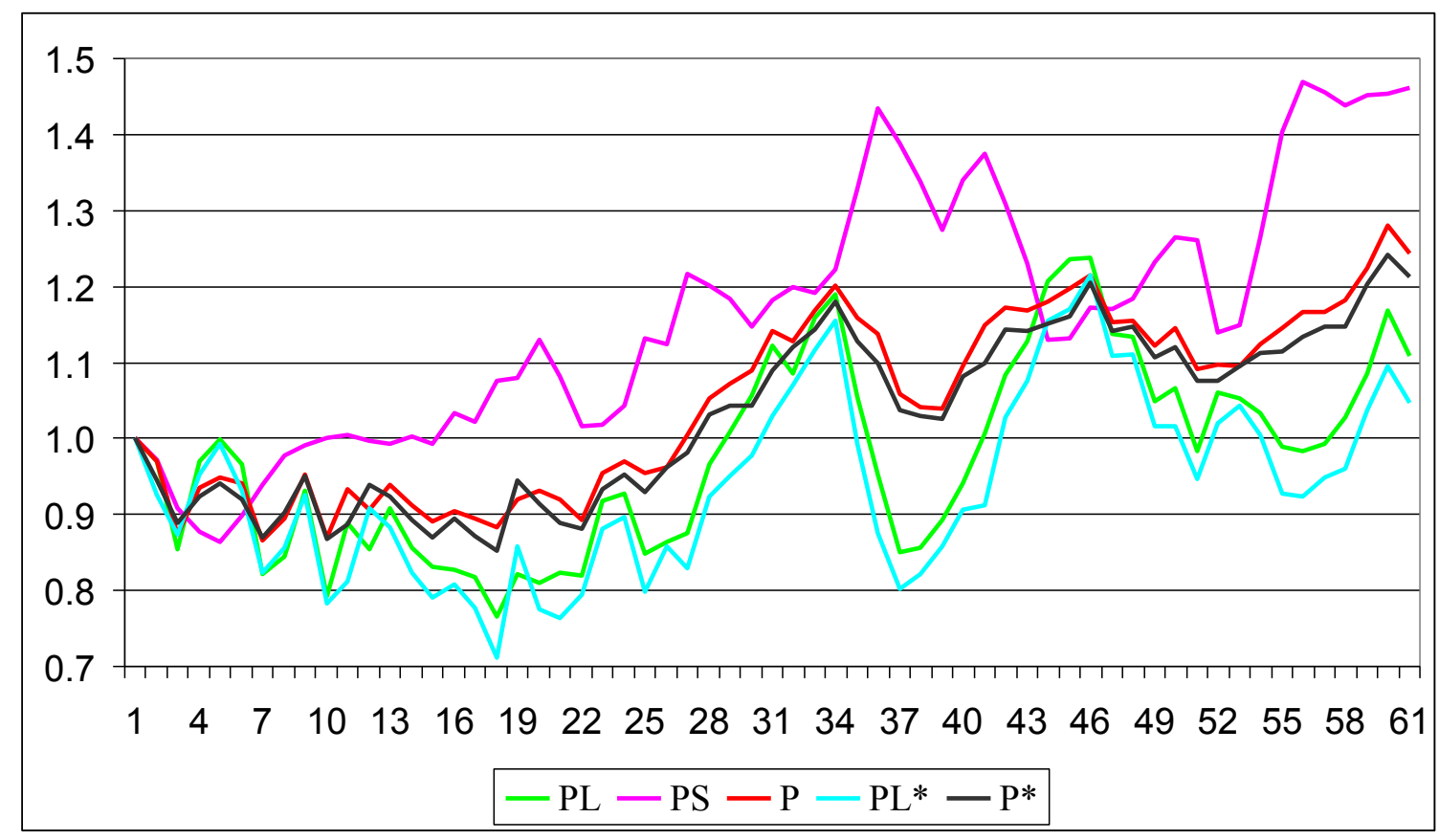

$\mathrm{PL}, \mathrm{PS}$ and $\mathrm{P}$ on Chart 1 correspond to the price indexes $\mathrm{p}_{\mathrm{Lt}}, \mathrm{p}_{\mathrm{St}}$ and $\mathrm{p}_{\mathrm{t}}$ defined in this section while $\mathrm{PL}^{*}$ and $\mathrm{P}^{*}$ correspond to the land and property price indexes that correspond to the section 13 model, $\mathrm{p}_{\mathrm{Lt}}{ }^{*}$ and $\mathrm{p}_{\mathrm{t}}{ }^{*}$, which will be defined shortly. Note that $\mathrm{p}_{\mathrm{St}}$ (PS on the chart) has some large increases in the middle and end of the sample period. These increases were driven by increasing construction costs due to earthquakes and other disasters. It can be seen that as construction prices spike upwards, land prices tend to spike downwards. It can also be seen that for the most part, the land price index is below the structure price index.

We turn now to the model defined in section 13 above. Once we have estimated the unknown parameters for this model ${ }^{36}$ we can form predicted land values for observations tn in rich, medium and poor wards, $\mathrm{V}_{\mathrm{LRtn}}, \mathrm{V}_{\mathrm{LMtn}}$ and $\mathrm{V}_{\mathrm{LPtn}}$ respectively, defined as follows: ${ }^{37}$

(35) $\mathrm{V}_{\mathrm{LRtn}}=\alpha_{\mathrm{tR}}{ }^{*}\left(\omega_{5}{ }^{*} \mathrm{D}_{\mathrm{W}, \mathrm{tn}, 5}+\omega_{6}{ }^{*} \mathrm{D}_{\mathrm{W}, \mathrm{tn}, 6}\right) \psi_{\mathrm{tn}}{ }^{*}$;

(36) $\mathrm{V}_{\mathrm{LMtn}}=\alpha_{\mathrm{tM}}{ }^{*}\left(\sum_{\mathrm{j}=1}{ }^{4} \omega_{\mathrm{j}}^{*} \mathrm{D}_{\mathrm{W}, \mathrm{tn}, \mathrm{j}}+\omega_{9}{ }^{*} \mathrm{D}_{\mathrm{W}, \mathrm{tn}, 9}\right) \psi_{\mathrm{tn}}{ }^{*}$;

(37) $\mathrm{V}_{\mathrm{LPtn}}=\alpha_{\mathrm{tP}}{ }^{*}\left(\omega_{7}^{*} \mathrm{D}_{\mathrm{W}, \mathrm{tn}, 7}+\omega_{8}^{*} \mathrm{D}_{\mathrm{W}, \mathrm{tn}, 8}\right) \psi_{\mathrm{tn}}{ }^{*}$

where $\psi_{\mathrm{tn}}{ }^{*}$ is defined as follows for $\mathrm{t}=1, \ldots, 61$ and $\mathrm{n}=1, \ldots, \mathrm{N}(\mathrm{t})$ :

(38) $\psi_{\mathrm{tn}}{ }^{*} \equiv\left(\sum_{\mathrm{h}=1}{ }^{14} \chi_{\mathrm{h}}{ }^{*} \mathrm{D}_{\mathrm{TH}, \mathrm{tn}, \mathrm{h}}\right)\left(\sum_{\mathrm{m}=1}{ }^{10} \mu_{\mathrm{m}}{ }^{*} \mathrm{D}_{\mathrm{EL}, \mathrm{tn}, \mathrm{m}}\right)\left(\phi_{1}{ }^{*} \mathrm{D}_{\mathrm{S}, \mathrm{tn}, 1}+\phi_{2}{ }^{*} \mathrm{D}_{\mathrm{S}, \mathrm{tn}, 2}\right)\left(1+\gamma^{*}\left(\mathrm{H}_{\mathrm{tn}}-3\right)\right)$ $\times\left(1+\kappa^{*}\left(\mathrm{~N}_{\mathrm{tn}}-11\right)\right)\left(1+\eta^{*}\left(\mathrm{TW}_{\mathrm{tn}}-1\right)\right)\left(1+\theta^{*}\left(\mathrm{TT}_{\mathrm{tn}}-12\right)\right) \mathrm{L}_{\mathrm{tn}}\left(\lambda^{*}\right)$.

\footnotetext{
${ }^{36}$ The estimated coefficients are listed in Table A1 in the Appendix.

${ }^{37}$ Again define $\alpha_{1}{ }^{*} \equiv 1, \chi_{1}{ }^{*} \equiv 1, \mu_{1}{ }^{*} \equiv 1, \phi_{1}{ }^{*} \equiv 1$ and $\rho_{1}{ }^{*} \equiv 1$.
} 
It appears that we are assigning property $\mathrm{n}$ in quarter $\mathrm{t}$ to rich, medium and poor wards simultaneously but the ward dummy variables will ensure that each property will be assigned to only one group; the assigned values to the other two groups will be equal to zero. Now sum up the predicted values for land in rich, medium and poor wards for each sale in the quarter $\mathrm{t}$ to form total predicted (or imputed) values for land in rich, medium and poor wards, $\mathrm{V}_{\mathrm{LRt}}, \mathrm{V}_{\mathrm{LMt}}$ and $\mathrm{V}_{\mathrm{LPt}}$, as follows:

$$
\mathrm{V}_{\mathrm{LRt}} \equiv \Sigma_{\mathrm{neN}(\mathrm{t})} \mathrm{V}_{\mathrm{LRtn}} ; \mathrm{V}_{\mathrm{LMt}} \equiv \Sigma_{\mathrm{n} \in \mathrm{N}(\mathrm{t})} \mathrm{V}_{\mathrm{LMtn}} ; \mathrm{V}_{\mathrm{LPt}} \equiv \Sigma_{\mathrm{neN}(\mathrm{t})} \mathrm{V}_{\mathrm{LPtn}} ; \quad \mathrm{t}=1, \ldots, 61
$$

The land price indexes for rich, medium and poor wards turn out to be the estimated $\alpha_{\mathrm{t}}{ }^{*}$, $\alpha_{\mathrm{tM}}{ }^{*}$ and $\alpha_{\mathrm{tP}}{ }^{*}$ and the corresponding imputed quantities of land sold in quarter $\mathrm{t}$ for the three types of ward are defined by the following counterparts to equations (33):

$$
\begin{aligned}
& \mathrm{p}_{\mathrm{LRt}} \equiv \alpha_{\mathrm{tR}}{ }^{*} ; \mathrm{q}_{\mathrm{LRt}} \equiv \mathrm{V}_{\mathrm{LRt}} / \mathrm{p}_{\mathrm{LRt}} ; \mathrm{p}_{\mathrm{LMt}} \equiv \alpha_{\mathrm{tM}}{ }^{*} ; \mathrm{q}_{\mathrm{LMt}} \equiv \mathrm{V}_{\mathrm{LMt}} / \mathrm{p}_{\mathrm{LMt}} ; \\
& \mathrm{p}_{\mathrm{LPt}} \equiv \alpha_{\mathrm{tP}}{ }^{*} ; \mathrm{q}_{\mathrm{LPt}} \equiv \mathrm{V}_{\mathrm{LPt}} / \mathrm{p}_{\mathrm{LPt}} ;
\end{aligned}
$$$$
\mathrm{t}=1, \ldots, 61 .
$$

A land price index $\mathrm{p}_{\mathrm{Lt}}{ }^{*}$ is formed as a chained Fisher index of the three ward land indexes and it is plotted on Charts 1 and $2 . .^{38}$

The imputed value of structures for sale $\mathrm{n}$ in quarter $\mathrm{t}, \mathrm{V}_{\mathrm{Stn}}$, is still defined by (31) (with the section 13 estimates for $\delta^{*}, \sigma^{*}$ and the $\rho_{\mathrm{i}}^{*}$ replacing the section 12 estimates), the total imputed value of structures sold in quarter $\mathrm{t}$ is $\mathrm{V}_{\mathrm{St}} \equiv \Sigma_{\mathrm{n} \in \mathrm{N}(\mathrm{t})} \mathrm{V}_{\mathrm{Stn}}$, the period t price index for structures is $p_{\mathrm{St}}$ (the same index as used in section 12) and the imputed quantity of structures sold in quarter $\mathrm{t}$ is $\mathrm{q}_{\mathrm{St}} \equiv \mathrm{V}_{\mathrm{St}} / \mathrm{p}_{\mathrm{St}}$ for $\mathrm{t}=1, \ldots, 61$.

Finally, an overall property sales price index $p_{t}{ }^{*}$ is formed as a chained Fisher index of the three ward land indexes and the structure index and it is plotted on Charts 1 and 2.

Chart 2: Land Price Indexes for Rich, Medium and Poorer Wards ( $p_{L R t}, p_{L M t}$ and $\left.p_{L P t}\right)$, Land Price Index $p_{L t}{ }^{*}$ and Overall Property Price Index $p_{t}{ }^{*}$ for the Section 13 Model

\footnotetext{
${ }^{38}$ Thus $\mathrm{p}_{\mathrm{Lt}}{ }^{*}$ is listed as $\mathrm{PL}^{*}$ on Charts 1 and 2 . The rich, medium, poor ward and overall land price indexes, $\mathrm{p}_{\mathrm{LRt}}, \mathrm{p}_{\mathrm{LMt}}, \mathrm{p}_{\mathrm{LPt}}$ and $\mathrm{p}_{\mathrm{Lt}}{ }^{*}$ are listed in Table $\mathrm{A} 3$ in the Appendix.
} 


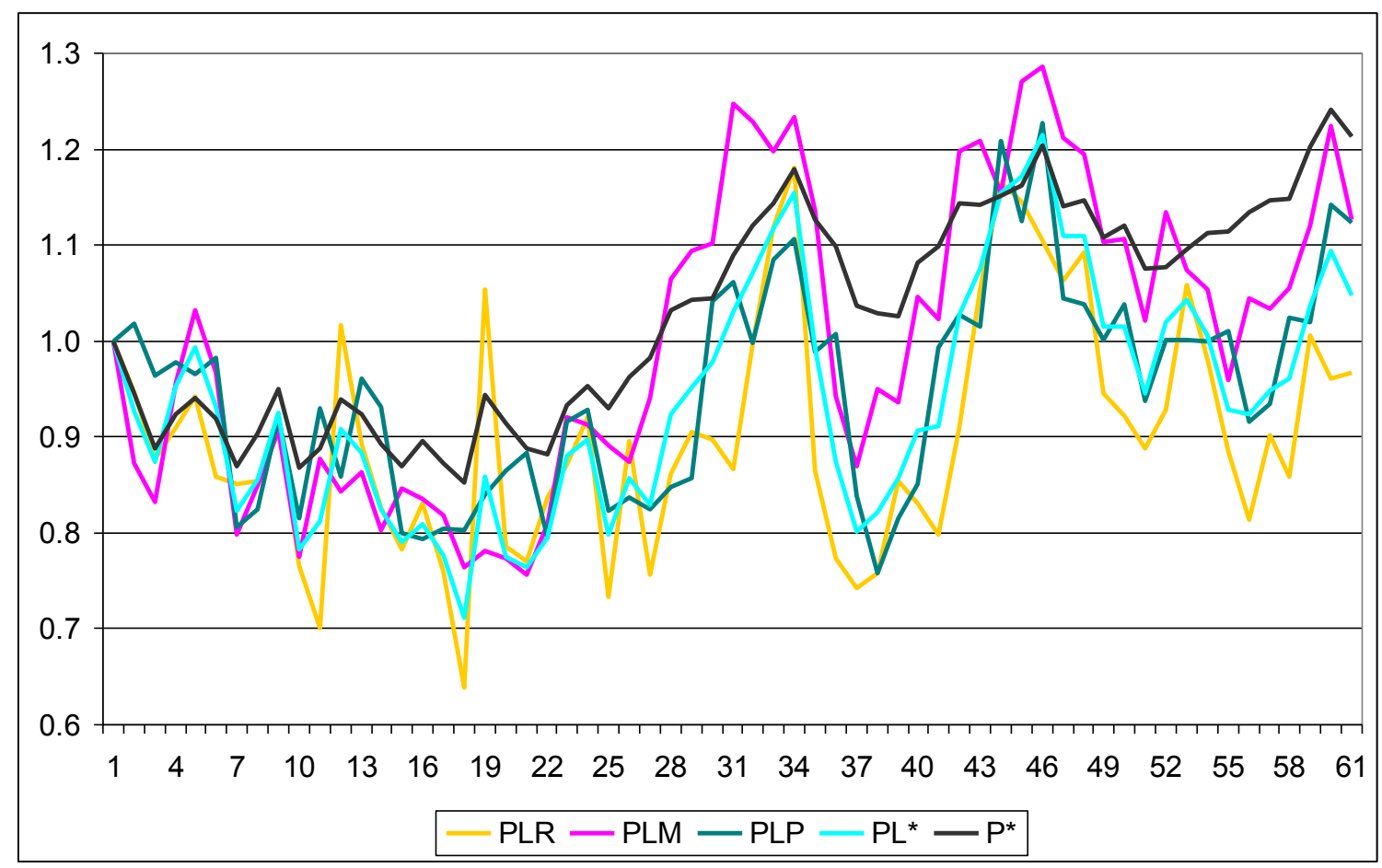

It can be seen that the land price index for rich wards is very erratic and hence it is likely that the model developed in section 13 is not reliable. There are not enough observations to reliably determine land prices by ward. ${ }^{39}$ Thus we regard the land price index and the overall condo sales price index that resulted from the hedonic regression model presented in section 12, $\mathrm{p}_{\mathrm{Lt}}$ and $\mathrm{p}_{\mathrm{t}}$, as our preferred price indexes for Tokyo condominium sales in the 9 Wards in our sample. ${ }^{40}$

In the following section, we will compare our preferred condo sales price index $\mathrm{p}_{\mathrm{t}}$ (based on the section 12 hedonic model) with other condo price indexes which have been suggested in the literature.

\section{Comparison of the Section 12 Price Index with other Condo Price Indexes}

The price indexes which we have been constructing are indexes for the sales of condominium units in a quarter. But for many purposes, there is more interest in having price indexes for the stock of condo units in a particular area. However, in order to form a stock index, it is necessary to have information on the stock of condo units in the areainformation which is generally not available. An approximation to the stock of condo units can be obtained by considering the total population of condo units that traded over the 61 quarters in our sample of property sales. Recall equations (33) and (34) in section 12 where the total quantity of condo land associated with the traded units in quarter $t$ was

\footnotetext{
${ }^{39}$ The minimum number of transactions in the 61 quarters was 23 . The number of transactions per quarter increased markedly towards the end of the sample period and so the our indexes are likely more reliable towards the end of the sample period.

${ }^{40}$ However, note that Chart 1 shows that there is not a great deal of difference between the overall condo price indexes based on the hedonic models presented in sections 12 and $13, \mathrm{p}_{\mathrm{t}}$ and $\mathrm{p}_{\mathrm{t}}{ }^{*}$.
} 
$\mathrm{q}_{\mathrm{Lt}} \equiv \mathrm{V}_{\mathrm{Lt}} / \mathrm{p}_{\mathrm{Lt}}$ and the total quantity of constant quality structures associated with the traded units in quarter $\mathrm{t}$ was $\mathrm{q}_{\mathrm{St}} \equiv \mathrm{V}_{\mathrm{St}} / \mathrm{p}_{\mathrm{St}}$ for $\mathrm{t}=1, \ldots, 61$. We approximate the population constant quality amounts of land and structures, $\mathrm{q}_{\mathrm{L}}$ and $\mathrm{q}_{\mathrm{S}}$, by summing up the $\mathrm{q}_{\mathrm{Lt}}$ and $\mathrm{q}_{\mathrm{St}}$ in our sample of condominium sales:

(41) $\mathrm{q}_{\mathrm{L}} \equiv \Sigma_{\mathrm{t}=1}{ }^{61} \mathrm{q}_{\mathrm{Lt}} ; \mathrm{q}_{\mathrm{S}} \equiv \Sigma_{\mathrm{t}=1}{ }^{61} \mathrm{q}_{\mathrm{St}}$.

We use the quantity weights defined by (41) along with the land and structure price indexes associated with the hedonic regression model defined in section $12, \mathrm{p}_{\mathrm{Lt}}$ and $\mathrm{p}_{\mathrm{St}}$, to define the following Lowe index ${ }^{41}$ for quarter $\mathrm{t}$ :

$$
\mathrm{p}_{\mathrm{LOWEt}} \equiv\left(\mathrm{p}_{\mathrm{Lt}} \mathrm{q}_{\mathrm{L}}+\mathrm{p}_{\mathrm{St}} \mathrm{q}_{\mathrm{S}}\right) /\left(\mathrm{p}_{\mathrm{L} 1} \mathrm{q}_{\mathrm{L}}+\mathrm{p}_{\mathrm{S} 1} \mathrm{q}_{\mathrm{S}}\right)
$$

The section 12 sales price index $\mathrm{p}_{\mathrm{t}}$ and the approximate stock price index $\mathrm{p}_{\mathrm{LOWEt}}$ are plotted on Chart 3. It can be seen that the two indexes cannot be distinguished from each other on the Chart except for a few quarters towards the end of the sample period. ${ }^{42}$

Chart 3 also plots the mean and median prices for the condo units sold in each quarter, $\mathrm{p}_{\text {MEANt }}$ and $\mathrm{p}_{\text {MEDt, }}$ where these series have been normalized to equal unity in quarter $1 .^{43}$ Somewhat surprisingly, the median series is more volatile than the mean series and both series are far more volatile than our preferred overall condo price indexes, $\mathrm{p}_{\mathrm{t}}$ and $\mathrm{p}_{\mathrm{LOWEt}}$. As might be expected, the mean and median series have a serious downward bias as compared to our preferred indexes due to the fact that means and medians in general do not adequately account for net depreciation of the structure component of condo prices. ${ }^{44}$

\section{Chart 3: The Overall Sales Price Index $p_{t}$, the Lowe Index $p_{\text {LowEt }}$, the Traditional Time Dummy Hedonic Regression Sales Price Index $\mathbf{p}_{\mathrm{TDt}}$ and the Quarterly Mean and Median Price Indexes of Sales, p Meant $_{\text {and }} \mathbf{p}_{\text {MEDt }}$}

\footnotetext{
${ }^{41}$ The Lowe index is a fixed basket index where the quantities remain fixed over time while the prices vary. It is named in honour of Lowe (1823) who introduced this index and is the father of the Consumer Price Index; see Hill (2004) for additional discussion. The approximate stock price index methodology that we introduce here was applied in the residential property context; see Eurostat $(2013 ; 98)$.

${ }^{42}$ This result is similar to the residential property comparison of similar indexes for a town in the Netherlands; see Eurostat $(2013 ; 98)$.

${ }^{43}$ To recover the sequence of mean and median prices in units of 10,000 yen in quarter $t$, multiply p pEANt by 2542.39 and multiply $p_{M E D t}$ by 2450.00 . Thus on average, the unadjusted mean prices lie above the corresponding median prices, as might be expected.

${ }^{44}$ In quarter $61, \mathrm{p}_{\mathrm{t}}$ and $\mathrm{p}_{\mathrm{LOWEt}}$ end up at 1.2437 and 1.2411 respectively while $\mathrm{p}_{\mathrm{MEANt}}$ and $\mathrm{p}_{\mathrm{MEDt}}$ end up at 1.1102 and 1.1347. In a steady state economy where the number of newly constructed condo units is offset by the demolition of old condo units, the mean and median indexes could adequately account for the net depreciation of condo units.
} 


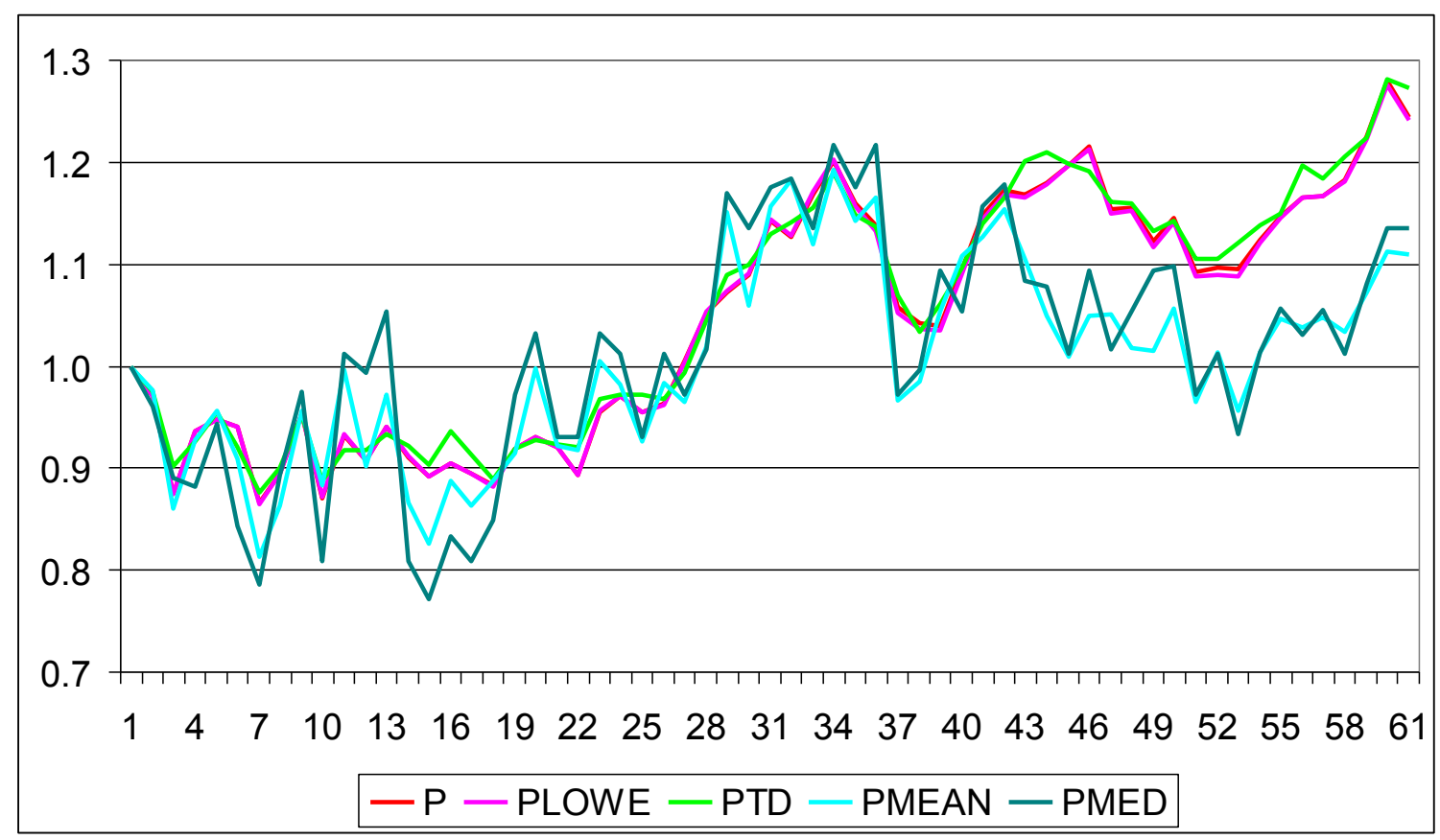

Chart 3 also plots a traditional time dummy hedonic price index ${ }^{45}$ for condo sales in our sample of properties, $\mathrm{p}_{\mathrm{TDt}}$. In order to construct this index, let $\ln \mathrm{V}_{\mathrm{tn}}, \ln \mathrm{S}_{\mathrm{tn}}$ and $\ln \mathrm{L}_{\mathrm{tn}}$ denote the natural logarithms of $\mathrm{V}_{\mathrm{tn}}, \mathrm{S}_{\mathrm{tn}}$ and $\mathrm{L}_{\mathrm{tn}}$ respectively where $\mathrm{L}_{\mathrm{tn}} \equiv\left(\mathrm{S}_{\mathrm{tn}} / \mathrm{TS}_{\mathrm{tn}}\right) \mathrm{TL}_{\mathrm{tn}}$. The 9

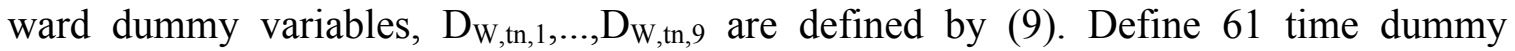
variables, $\mathrm{D}_{\mathrm{T}, \mathrm{tn}, 1}, \ldots, \mathrm{D}_{\mathrm{T}, \mathrm{tn}, 61}$ in an analogous manner. Now run a linear regression with a constant term with the $\ln \mathrm{V}_{\text {tn }}$ as the dependent variables and the following variables as

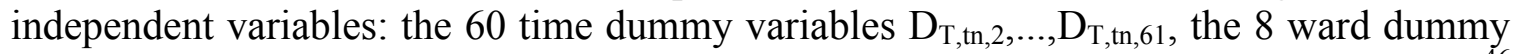

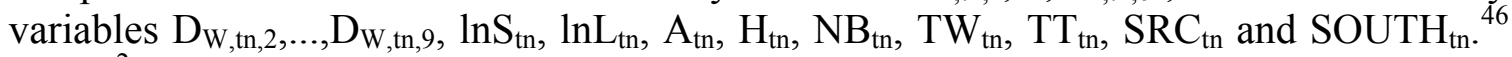
The $\mathrm{R}^{2}$ for this regression turned out to be 0.8787 which is a very satisfactory fit. The time dummy hedonic price index $\mathrm{p}_{\mathrm{TDt}}$ was constructed as follows: $\equiv 1$; for quarters $\mathrm{t}=$ $2, \ldots, 61$, define $\mathrm{p}_{\mathrm{TDt}}$ as the exponential of the estimated coefficient for the time dummy variable $\mathrm{D}_{\mathrm{T}, \mathrm{tn}, \mathrm{t}}$. The resulting index $\mathrm{p}_{\mathrm{TDt}}$ is also plotted on Chart 3 . It can be seen that $\mathrm{p}_{\mathrm{TDt}}$ is very close to our preferred overall condo price indexes, $p_{t}$ and $p_{\text {LOWEt }}{ }^{47}$ The five indexes plotted on Chart 3 are listed in Table A4 of the Appendix.

\footnotetext{
${ }^{45}$ This type of hedonic index also dates back to Court (1939).

${ }^{46}$ Here are the estimated coefficients for the constant term, $\mathrm{D}_{\mathrm{W}, \mathrm{nn}, 2}, \ldots, \mathrm{D}_{\mathrm{W}, \mathrm{tn}, 9}, \ln \mathrm{S}_{\mathrm{tn}}, \ln \mathrm{L}_{\mathrm{tn}}, \mathrm{A}_{\mathrm{tn}}, \mathrm{H}_{\mathrm{tn}}, \mathrm{NB}_{\mathrm{tn}}$, $\mathrm{TW}_{\mathrm{tn}}, \mathrm{TT}_{\mathrm{tn}}, \mathrm{SRC}_{\mathrm{tn}}$ and $\mathrm{SOUTH}_{\mathrm{tn}}$ with t statistics in brackets: $4.4850(7147), 0.0049(0.559), 0.0185(1.417)$, -0.0798 (-7.309), 0.0965 (8.840), 0.2475 (20.52), -0.1819 (-19.04), -0.1147 (-11.75), -0.0224 (-2.341), 0.9158 (53.86), 0.0203 (3.062), -0.0168 (-59.74), 0.0076 (9.536), $0.0034(0.870),-0.0135$ (-32.40), $-0.0094(-25.78),-0.0027(-0.6292)$ and $0.0195(2.983)$. The signs of the estimated coefficients are all as expected with the exception of the reinforced concrete coefficient which was slightly negative $(-0.0027)$ but statistically insignificant. The magnitudes of the estimated coefficients for the logs of structures and land were 0.9158 and 0.0203 which gives a very large weight to the structure area of the condo and a very small weight to its imputed land area. The age coefficient was -0.0168 which means that the estimated annual net depreciation rate as a percentage of property value was a very reasonable $1.68 \%$ per year.

${ }^{47}$ The correlation coefficients of $\mathrm{p}_{\mathrm{t}}$ with $\mathrm{p}_{\mathrm{LOWEt}}, \mathrm{p}_{\mathrm{TD}}$, $\mathrm{p}_{\text {MEANt }}$ and $\mathrm{p}_{\text {MEDt }}$ were $0.9998,0.9933,0.8294$ and 0.7765 respectively.
} 
The close correspondence of the time dummy hedonic index $\mathrm{p}_{\mathrm{TDt}}$ with our preferred condo sales index $p_{t}$ that was described in section 12 is of some interest. It is much easier to run a simple linear regression than to run a sequence of nonlinear regressions where each model gradually becomes more complex. However, the simple linear regression approach can sometimes have multicollinearity problems which generate implausible parameter estimates. Thus the simple model closely approximates the more complex model in this case because both models generate reasonably sensible parameter estimates. The simple time dummy approach does not always work well. In particular, in evaluating the plausibility of the simple time dummy approach, attention should be paid to the sign and magnitude of the age coefficient. If the estimated age coefficient turns out to be large and positive, the resulting time dummy price index is likely to have a substantial downward bias and so the resulting index should be viewed with caution. ${ }^{48}$

\section{Conclusion}

Determining the main characteristics of a condominium property that determine its selling price is a much more difficult task than the determination of relevant characteristics for detached dwelling units. Apartment complexes have height characteristics as well as length and width characteristics which affect prices. This paper provides a starting point for determining these relevant characteristics. Some of the characteristics which we found to be price determining (in addition to the usual important characteristics like the floor space of the unit, the land area of the building, the location of the building and the age of the building) were the height of the sold unit, the height of the building, the number of units in the building and the amount of excess land on the property.

The main focus of our paper has been to suggest a method for decomposing condominium values into structure and land components, where the structure value components are consistent with existing national statistical agency practices for the determination of apartment structure values.

The following points were made during the course of the paper:

- Our method led to an estimated geometric depreciation rate for Tokyo apartment buildings of about $3.6 \%$ per year, which seems reasonable. ${ }^{49}$

\footnotetext{
${ }^{48}$ Diewert and Shimizu (2016) implemented a nonlinear regression model for commercial properties in Tokyo that is similar in many respects to our present model for condos and they compared their preferred commercial property price index with a simple time dummy hedonic regression model. They found a substantial downward bias in the time dummy index due to the fact that the coefficient on age in the time dummy regression was large and positive.

${ }^{49}$ Official Bureau of Economic Analysis geometric depreciation rates for US residential and commercial structures range from $2.3 \%$ to $3.2 \%$ per year. A recent study by Geltner and Bokhari (2015) estimates structure net depreciation for apartments at 3.9\% per year. Note that our estimated structure depreciation rate applies to condo units in buildings which have not been demolished. The additional depreciation due to the demolition of apartment blocks needs to be added to our estimated depreciation rate. Diewert and Shimizu (2016) found that this additional demolition depreciation rate was approximately $2 \%$ per year for Tokyo commercial office buildings and it is likely that a similar rate applies to apartment blocks.
} 
- Our preferred overall price index for condo sales was virtually identical to the corresponding Lowe index which provides an approximation to a price index for the stock of condo units in Tokyo. These indexes were substantially smoother and generally above the corresponding indexes based on the means and medians of condo sales in each quarter. The latter indexes tend to have a downward bias due to their neglect of net depreciation of the structure.

- Traditional time dummy hedonic regressions can generate reasonable overall price indexes for condo sales. However, one should check whether the estimated coefficient for the age of the structure is negative and of a reasonable magnitude. If the estimated coefficient is large and positive, the resulting time dummy price index is likely to have a substantial downward bias.

The results generated in this paper should be regarded as provisional. Our models need to be tested on other data sets and improvements to our basic methodology should be made. However, the models presented in this paper provide a starting point for further research. Moreover, many of the explanatory characteristics that were used to explain the variation in the value of condominium properties will probably be useful in explaining commercial property prices.

\section{Appendix}

Table A1 lists the estimated coefficients for the model described in section 13 of the paper.

Table A1: Estimated Coefficients for the Section 13 Hedonic Model

\begin{tabular}{|c|c|c|c|c|c|c|c|c|c|c|c|}
\hline Coef & Estimate & t stat & Coef & Estimate & t stat & Coef & Estimate & t stat & Coef & Estimate & t stat \\
\hline$\omega_{5}{ }^{*}$ & 76.57 & 12.71 & $\alpha_{55 \mathrm{R}}{ }^{*}$ & 1.0103 & 14.04 & $\alpha_{17 \mathrm{M}^{*}}$ & 0.8179 & 13.44 & $\alpha_{10 \mathrm{P}}{ }^{*}$ & 0.7660 & 3.83 \\
\hline$\omega_{6}{ }^{*}$ & 102.31 & 12.69 & $\alpha_{56 \mathrm{R}}{ }^{*}$ & 0.9157 & 13.83 & $\alpha_{18 \mathrm{M}}{ }^{*}$ & 0.7649 & 13.51 & $\alpha_{11 \mathrm{P}}{ }^{*}$ & 0.7000 & 5.05 \\
\hline$\alpha_{2 \mathrm{R}}{ }^{*}$ & 1.0185 & 13.19 & $\alpha_{57 \mathrm{R}}{ }^{*}$ & 0.9345 & 13.53 & $\alpha_{19 \mathrm{M}}{ }^{*}$ & 0.7805 & 12.78 & $\alpha_{12 \mathrm{P}}{ }^{*}$ & 1.0170 & 5.17 \\
\hline$\alpha_{3 R^{*}}$ & 0.9643 & 8.45 & $\alpha_{58 \mathrm{R}}{ }^{*}$ & 1.0239 & 14.28 & $\alpha_{20 M}{ }^{*}$ & 0.7738 & 15.97 & $\alpha_{13 \mathrm{P}}{ }^{*}$ & 0.8931 & 4.86 \\
\hline$\alpha_{4 R^{*}}$ & 0.9774 & 12.99 & $\alpha_{59 R}{ }^{*}$ & 1.0201 & 13.22 & $\alpha_{21 \mathrm{M}}{ }^{*}$ & 0.7564 & 14.54 & $\alpha_{14 \mathrm{P}}{ }^{*}$ & 0.8257 & 6.46 \\
\hline$\alpha_{5 R^{*}}$ & 0.9648 & 13.67 & $\alpha_{60 \mathrm{R}}{ }^{*}$ & 1.1422 & 14.27 & $\alpha_{22 \mathrm{M}}{ }^{*}$ & 0.8086 & 14.21 & $\alpha_{15 \mathrm{P}}{ }^{*}$ & 0.7828 & 6.81 \\
\hline$\alpha_{6 R^{*}}$ & 0.9821 & 11.85 & $\alpha_{61 R^{*}}$ & 1.1238 & 14.10 & $\alpha_{23 \mathrm{M}}{ }^{*}$ & 0.9202 & 17.92 & $\alpha_{16 \mathrm{P}}{ }^{*}$ & 0.8300 & 6.97 \\
\hline$\alpha_{7 \mathrm{R}}{ }^{*}$ & 0.8052 & 11.25 & $\chi_{2}^{*}$ & 1.2771 & 19.70 & $\alpha_{24 \mathrm{M}}{ }^{*}$ & 0.9128 & 17.66 & $\alpha_{17 \mathrm{P}}{ }^{*}$ & 0.7600 & 8.19 \\
\hline$\alpha_{8 \mathrm{R}}{ }^{*}$ & 0.8241 & 9.69 & $\chi_{3}{ }^{*}$ & 1.3538 & 21.62 & $\alpha_{25 \mathrm{M}}{ }^{*}$ & 0.8907 & 16.67 & $\alpha_{18 \mathrm{P}}{ }^{*}$ & 0.6392 & 5.70 \\
\hline$\alpha_{9 \mathrm{R}}{ }^{*}$ & 0.9212 & 13.15 & $\chi_{4}{ }^{*}$ & 1.6103 & 21.59 & $\alpha_{26 M^{*}}$ & 0.8740 & 18.02 & $\alpha_{19 \mathrm{P}}{ }^{*}$ & 1.0532 & 5.88 \\
\hline$\alpha_{10 R^{*}}$ & 0.8150 & 11.72 & $\chi_{5}{ }^{*}$ & 1.7248 & 21.84 & $\alpha_{27 M^{*}}$ & 0.9402 & 15.00 & $\alpha_{20 \mathrm{P}}{ }^{*}$ & 0.7862 & 7.53 \\
\hline$\alpha_{11 R}{ }^{*}$ & 0.9298 & 11.62 & $\chi_{6}{ }^{*}$ & 1.8488 & 21.68 & $\alpha_{28 M}{ }^{*}$ & 1.0644 & 17.45 & $\alpha_{21 \mathrm{P}}{ }^{*}$ & 0.7697 & 6.70 \\
\hline$\alpha_{12 R}{ }^{*}$ & 0.8590 & 10.15 & $\chi_{7}{ }^{*}$ & 1.9044 & 21.25 & $\alpha_{29 \mathrm{M}}{ }^{*}$ & 1.0934 & 21.17 & $\alpha_{22 \mathrm{P}}{ }^{*}$ & 0.8374 & 7.25 \\
\hline$\alpha_{13 \mathrm{R}}{ }^{*}$ & 0.9614 & 12.23 & $\chi_{8}{ }^{*}$ & 1.9527 & 21.16 & $\alpha_{30 \mathrm{M}}{ }^{*}$ & $\mathbf{1 . 1 0 1 5}$ & 19.19 & $\alpha_{23 \mathrm{P}}{ }^{*}$ & 0.8713 & 7.90 \\
\hline$\alpha_{14 \mathrm{R}}{ }^{*}$ & 0.9320 & 10.74 & $\chi_{9}{ }^{*}$ & 1.9172 & 20.82 & $\alpha_{31 \mathrm{M}}{ }^{*}$ & 1.2466 & 18.06 & $\alpha_{24 \mathrm{P}}{ }^{*}$ & 0.9203 & 8.81 \\
\hline$\alpha_{15 \mathrm{R}}{ }^{*}$ & 0.7996 & 10.56 & $\chi_{10}{ }^{*}$ & 2.0205 & 20.36 & $\alpha_{32 \mathrm{M}}{ }^{*}$ & 1.2283 & 17.85 & $\alpha_{25 \mathrm{P}}{ }^{*}$ & 0.7333 & 8.66 \\
\hline$\alpha_{16 \mathrm{R}}{ }^{*}$ & 0.7936 & 11.84 & $\chi_{11}{ }^{*}$ & 2.2598 & 20.16 & $\alpha_{33 \mathrm{M}}{ }^{*}$ & 1.1980 & 21.10 & $\alpha_{26 \mathrm{P}}{ }^{*}$ & 0.8966 & 7.12 \\
\hline$\alpha_{17 \mathrm{R}}{ }^{*}$ & 0.8051 & 11.45 & $\chi_{12}{ }^{*}$ & 2.1703 & 20.23 & $\alpha_{34 \mathrm{M}}{ }^{*}$ & 1.2333 & 21.40 & $\alpha_{27 \mathrm{P}}{ }^{*}$ & 0.7566 & 6.83 \\
\hline$\alpha_{18 R}{ }^{*}$ & 0.8036 & 10.20 & $\chi_{13}{ }^{*}$ & 2.3463 & 19.30 & $\alpha_{35 \mathrm{M}}{ }^{*}$ & 1.1356 & 20.39 & $\alpha_{28 \mathrm{P}}{ }^{*}$ & 0.8623 & 8.31 \\
\hline$\alpha_{19 R^{*}}$ & 0.8394 & 10.23 & $\chi_{14}{ }^{*}$ & 2.9628 & 16.66 & $\alpha_{36 M^{*}}$ & 0.9415 & 19.08 & $\alpha_{29 \mathrm{P}}{ }^{*}$ & 0.9049 & 6.54 \\
\hline$\alpha_{20 R}{ }^{*}$ & 0.8645 & 11.83 & $\gamma^{*}$ & 0.0161 & 8.66 & $\alpha_{37 M^{*}}$ & 0.8688 & 13.62 & $\alpha_{30 \mathrm{P}}{ }^{*}$ & 0.8980 & 8.49 \\
\hline$\alpha_{21 \mathrm{R}}{ }^{*}$ & 0.8839 & 12.59 & $\boldsymbol{K}^{*}$ & 0.0168 & 19.15 & $\alpha_{38 \mathrm{M}}{ }^{*}$ & 0.9504 & 17.03 & $\alpha_{31 \mathrm{P}}{ }^{*}$ & 0.8660 & 8.13 \\
\hline$\alpha_{22 \mathrm{R}}{ }^{*}$ & 0.7958 & 9.95 & $\mu_{2}{ }^{*}$ & 0.7449 & $\begin{array}{l}54.58 \\
\end{array}$ & $\alpha_{39 \mathrm{M}}{ }^{*}$ & 0.9359 & 17.50 & $\alpha_{32 \mathrm{P}}{ }^{*}$ & 0.9957 & 8.51 \\
\hline$\alpha_{23 \mathrm{R}}{ }^{*}$ & 0.9158 & 13.20 & $\mu_{3}{ }^{*}$ & 0.5978 & 52.58 & $\boldsymbol{\alpha}_{40 \mathrm{M}}{ }^{*}$ & 1.0456 & 15.55 & $\alpha_{33 \mathrm{P}}{ }^{*}$ & 1.1186 & 9.37 \\
\hline$\alpha_{24 \mathrm{R}^{*}}$ & 0.9288 & 12.81 & $\mu_{4}{ }^{*}$ & 0.4978 & 46.91 & $\alpha_{41 \mathrm{M}}{ }^{*}$ & 1.0235 & 13.99 & $\alpha_{34 \mathrm{P}}{ }^{*}$ & 1.1808 & 10.10 \\
\hline$\alpha_{25 R^{*}}$ & 0.8230 & 12.59 & $\mu_{5}{ }^{*}$ & 0.4509 & 43.75 & $\alpha_{42 \mathrm{M}}{ }^{*}$ & 1.1981 & 18.11 & $\alpha_{35 \mathrm{P}}{ }^{*}$ & 0.8645 & 8.92 \\
\hline$\alpha_{26 R^{*}}$ & 0.8373 & 12.66 & $\mu_{6}{ }^{*}$ & 0.4107 & 39.15 & $\alpha_{43 M^{*}}$ & 1.2094 & 18.86 & $\alpha_{36 \mathrm{P}}{ }^{*}$ & 0.7733 & 8.18 \\
\hline
\end{tabular}




\begin{tabular}{|c|c|c|c|c|c|c|c|c|c|c|c|}
\hline$\alpha_{27 R}{ }^{*}$ & 0.8240 & 11.53 & $\mu_{7}{ }^{*}$ & 0.3450 & 38.00 & $\alpha_{44 M}{ }^{*}$ & 1.1548 & 16.16 & $\alpha_{37 \mathrm{P}}{ }^{*}$ & 0.7419 & 7.40 \\
\hline$\alpha_{28 R}{ }^{*}$ & 0.8482 & 11.21 & $\mu_{8}{ }^{*}$ & 0.2980 & 37.16 & $\alpha_{45 M}{ }^{*}$ & 1.2703 & 19.18 & $\alpha_{38 \mathrm{P}}{ }^{*}$ & 0.7582 & 7.35 \\
\hline$\alpha_{29 R}{ }^{*}$ & 0.8571 & 12.09 & $\mu_{9}{ }^{*}$ & 0.2501 & 32.21 & $\alpha_{46 \mathrm{M}}{ }^{*}$ & 1.2861 & 19.32 & $\alpha_{39 \mathrm{P}}{ }^{*}$ & 0.8543 & 8.42 \\
\hline$\alpha_{30 \mathrm{R}}{ }^{*}$ & 1.0407 & 13.06 & $\mu_{10}{ }^{*}$ & 0.2170 & 24.59 & $\alpha_{47 \mathrm{M}}{ }^{*}$ & 1.2114 & 18.19 & $\alpha_{40 \mathrm{P}}{ }^{*}$ & 0.8309 & 8.60 \\
\hline$\alpha_{31 \mathrm{R}}{ }^{*}$ & 1.0611 & 12.76 & $\lambda^{*}$ & 0.4510 & 19.78 & $\alpha_{48 \mathrm{M}}{ }^{*}$ & 1.1953 & 19.89 & $\alpha_{41 \mathrm{P}}{ }^{*}$ & 0.7984 & 6.30 \\
\hline$\alpha_{32 \mathrm{R}}{ }^{*}$ & 0.9979 & 13.87 & $\phi_{2}{ }^{*}$ & 1.0272 & 134.83 & $\alpha_{49 \mathrm{M}}{ }^{*}$ & 1.1040 & 19.59 & $\alpha_{42 P}{ }^{*}$ & 0.9103 & 9.53 \\
\hline$\alpha_{33 \mathrm{R}}{ }^{*}$ & 1.0852 & 12.16 & $\eta^{*}$ & -0.0189 & -29.88 & $\alpha_{50 \mathrm{M}}{ }^{*}$ & 1.1066 & 21.03 & $\alpha_{43 \mathrm{P}}{ }^{*}$ & 1.0537 & 10.06 \\
\hline$\alpha_{34 \mathrm{R}}{ }^{*}$ & 1.1066 & 13.86 & $\theta^{*}$ & -0.0133 & -30.26 & $\alpha_{51 \mathrm{M}}{ }^{*}$ & 1.0214 & 20.08 & $\alpha_{44 \mathrm{P}}{ }^{*}$ & 1.1651 & 9.58 \\
\hline$\alpha_{35 \mathrm{R}}{ }^{*}$ & 0.9887 & 12.36 & $\omega_{1}{ }^{*}$ & 64.72 & 15.83 & $\alpha_{52 M}{ }^{*}$ & 1.1346 & 22.22 & $\alpha_{45 P}{ }^{*}$ & 1.1451 & 9.88 \\
\hline$\alpha_{36 R}{ }^{*}$ & 1.0070 & 13.24 & $\omega_{2}{ }^{*}$ & 63.04 & 15.79 & $\alpha_{53 \mathrm{M}}{ }^{*}$ & $\mathbf{1 . 0 7 3 7}$ & 21.26 & $\alpha_{46 P}{ }^{*}$ & 1.1045 & 10.21 \\
\hline$\alpha_{37 \mathrm{R}}{ }^{*}$ & 0.8391 & 10.94 & $\omega_{3}{ }^{*}$ & 65.03 & 15.48 & $\alpha_{54 \mathrm{M}}{ }^{*}$ & 1.0544 & 22.28 & $\alpha_{47 P}{ }^{*}$ & 1.0628 & 9.59 \\
\hline$\alpha_{38 \mathrm{R}}{ }^{*}$ & 0.7573 & 11.81 & $\omega_{4}{ }^{*}$ & 56.25 & 15.57 & $\alpha_{55 \mathrm{M}}{ }^{*}$ & 0.9593 & 19.68 & $\alpha_{48 \mathrm{P}}{ }^{*}$ & $\mathbf{1 . 0 9 3 1}$ & 10.63 \\
\hline$\alpha_{39 \mathrm{R}}{ }^{*}$ & 0.8145 & 10.98 & $\omega_{9}{ }^{*}$ & 59.37 & 15.66 & $\alpha_{56 \mathrm{M}}{ }^{*}$ & 1.0451 & 22.33 & $\alpha_{49 \mathrm{P}}{ }^{*}$ & 0.9448 & 10.15 \\
\hline$\alpha_{40 \mathrm{R}}{ }^{*}$ & 0.8511 & 11.50 & $\alpha_{2 M}{ }^{*}$ & 0.8724 & 14.11 & $\alpha_{57 M}{ }^{*}$ & 1.0337 & 21.82 & $\alpha_{50 \mathrm{P}}{ }^{*}$ & 0.9218 & 9.84 \\
\hline$\alpha_{41 \mathrm{R}}{ }^{*}$ & 0.9928 & 13.39 & $\alpha_{3 M}{ }^{*}$ & $\mathbf{0 . 8 3 2 7}$ & 14.56 & $\alpha_{58 M}{ }^{*}$ & 1.0550 & 21.34 & $\alpha_{51 \mathrm{P}}{ }^{*}$ & 0.8886 & 10.12 \\
\hline$\alpha_{42 R}{ }^{*}$ & 1.0279 & 13.18 & $\alpha_{4 M}{ }^{*}$ & 0.9559 & 16.60 & $\alpha_{59 M}{ }^{*}$ & 1.1200 & 21.96 & $\alpha_{52 \mathrm{P}}{ }^{*}$ & 0.9279 & 10.26 \\
\hline$\alpha_{43 \mathrm{R}}{ }^{*}$ & 1.0157 & 12.67 & $\alpha_{5 M}{ }^{*}$ & 1.0323 & 17.31 & $\alpha_{60 \mathrm{M}}{ }^{*}$ & 1.2238 & 22.04 & $\alpha_{53 \mathrm{P}}{ }^{*}$ & 1.0584 & 9.81 \\
\hline$\alpha_{44 \mathrm{R}}{ }^{*}$ & 1.2081 & 13.30 & $\alpha_{6 M}{ }^{*}$ & 0.9666 & 15.38 & $\alpha_{61 \mathrm{M}}{ }^{*}$ & 1.1271 & 22.80 & $\alpha_{54 \mathrm{P}}{ }^{*}$ & 0.9809 & 9.97 \\
\hline$\alpha_{45 \mathrm{R}}{ }^{*}$ & 1.1246 & 6.48 & $\alpha_{7 \mathrm{M}}{ }^{*}$ & 0.7976 & 13.96 & $\omega_{7}{ }^{*}$ & 48.01 & 10.33 & $\alpha_{55 \mathrm{P}}{ }^{*}$ & 0.8854 & 9.24 \\
\hline$\alpha_{46 \mathrm{R}}{ }^{*}$ & 1.2279 & 10.67 & $\alpha_{8 \mathrm{M}}{ }^{*}$ & 0.8497 & 17.45 & $\omega_{8}{ }^{*}$ & 54.84 & 10.40 & $\alpha_{56 \mathrm{P}}{ }^{*}$ & 0.8130 & 9.38 \\
\hline$\alpha_{47 \mathrm{R}}{ }^{*}$ & 1.0448 & 12.70 & $\alpha_{9 M}{ }^{*}$ & 0.9074 & 15.18 & $\alpha_{2 \mathrm{P}}{ }^{*}$ & 0.9469 & 8.02 & $\alpha_{57 \mathrm{P}}{ }^{*}$ & 0.9027 & 9.15 \\
\hline$\alpha_{48 R}{ }^{*}$ & 1.0376 & 13.98 & $\alpha_{10 M}{ }^{*}$ & 0.7749 & 15.03 & $\alpha_{3 \mathrm{P}}{ }^{*}$ & 0.8774 & 5.72 & $\alpha_{58 \mathrm{P}}{ }^{*}$ & 0.8590 & 9.77 \\
\hline$\alpha_{49 \mathrm{R}}{ }^{*}$ & 1.0016 & 14.03 & $\alpha_{11 M}{ }^{*}$ & 0.8776 & 13.90 & $\alpha_{4 \mathrm{P}}{ }^{*}$ & 0.9095 & 8.82 & $\alpha_{59 \mathrm{P}}{ }^{*}$ & 1.0054 & 9.21 \\
\hline$\alpha_{50 R^{*}}$ & 1.0375 & 14.18 & $\alpha_{12 M}{ }^{*}$ & 0.8429 & 14.08 & $\alpha_{5 \mathrm{P}}{ }^{*}$ & 0.9420 & 8.92 & $\alpha_{60 \mathrm{P}}{ }^{*}$ & 0.9607 & 9.64 \\
\hline$\alpha_{51 \mathrm{R}}{ }^{*}$ & 0.9383 & 13.59 & $\alpha_{13 \mathrm{M}}{ }^{*}$ & 0.8638 & 15.09 & $\alpha_{6 \mathrm{P}}{ }^{*}$ & 0.8586 & 6.66 & $\alpha_{61 \mathrm{P}}{ }^{*}$ & 0.9669 & 10.12 \\
\hline$\alpha_{52 \mathrm{R}}{ }^{*}$ & 1.0005 & 14.52 & $\alpha_{14 M}{ }^{*}$ & 0.8031 & 13.26 & $\alpha_{7 \mathrm{P}}{ }^{*}$ & $\mathbf{0 . 8 5 0 7}$ & 8.00 & $\delta^{*}$ & 0.0349 & 28.23 \\
\hline$\alpha_{53 \mathrm{R}}{ }^{*}$ & 1.0013 & 14.16 & $\alpha_{15 M}{ }^{*}$ & 0.8470 & 8.08 & $\alpha_{8 \mathrm{P}}{ }^{*}$ & 0.8544 & 7.94 & $\sigma^{*}$ & $\mathbf{0 . 0 3 5 7}$ & 2.65 \\
\hline \multirow[t]{2}{*}{$\alpha_{54 \mathrm{R}}{ }^{*}$} & 1.0001 & 14.51 & $\alpha_{16 M}{ }^{*}$ & 0.8348 & 13.10 & $\alpha_{9 \mathrm{P}}{ }^{*}$ & 0.9231 & 8.41 & $\rho_{2}{ }^{*}$ & 1.1462 & 106.72 \\
\hline & & & & & & & & & $\rho_{3}{ }^{*}$ & 1.2574 & 61.21 \\
\hline
\end{tabular}

Table A2 lists the land, structure and overall condo sales price indexes for each quarter $t$, $\mathrm{p}_{\mathrm{Lt}}, \mathrm{p}_{\mathrm{St}}{ }^{50}$ and $\mathrm{p}_{\mathrm{t}}$, that are generated by the hedonic regression model described in section 12. Note that the structure price index is the official Tokyo construction price index (normalized to equal 1 in the first quarter) and this index does not change across models. The land and structure shares of imputed property values in quarter $t$ for the section 12 model are defined as $\mathrm{s}_{\mathrm{Lt}} \equiv \mathrm{V}_{\mathrm{Lt}} /\left(\mathrm{V}_{\mathrm{Lt}}+\mathrm{V}_{\mathrm{St}}\right)$ and $\mathrm{s}_{\mathrm{St}} \equiv \mathrm{V}_{\mathrm{St}} /\left(\mathrm{V}_{\mathrm{Lt}}+\mathrm{V}_{\mathrm{St}}\right)$ where the $\mathrm{V}_{\mathrm{Lt}}$ and $\mathrm{V}_{\mathrm{St}}$ are defined by (32). These shares are also listed in Table A2. The sample average land share was 0.5797 and the sample average structure share of property value was 0.4203 . It can be seen that there is little long run trend in these shares.

Table A2: Land Prices $p_{L t}$, Structure Prices $p_{S t}$ and Overall Condo Prices $p_{t}$; Land and Structure Shares of Property Values, $s_{L t}$ and $s_{S t}$, and Constant Quality Land and Structure Quantities, $q_{L t}$ and $q_{S t}$ for the Section 12 Hedonic Model

\begin{tabular}{|c|c|c|c|c|c|c|c|}
\hline Quarter t & $\mathbf{p}_{\mathrm{Lt}}$ & $\mathbf{p}_{\mathrm{st}}$ & $\mathbf{p}_{\mathrm{t}}$ & $\mathbf{S}_{\mathbf{L t}}$ & $\mathbf{S}_{\mathrm{St}}$ & $\mathbf{q}_{\mathbf{L t}}$ & $\mathbf{q}_{\mathrm{s}}$ \\
\hline 1 & 1.0000 & 1.0000 & 1.0000 & 0.5708 & 0.4292 & 59022.5 & 44378.8 \\
\hline 2 & 0.9703 & 0.9714 & 0.9708 & 0.6163 & $\mathbf{0 . 3 8 3 7}$ & 57703.9 & 35881.3 \\
\hline 3 & 0.8540 & 0.9073 & 0.8740 & 0.6181 & 0.3819 & 36421.4 & 21178.8 \\
\hline 4 & 0.9707 & 0.8782 & 0.9360 & 0.6402 & 0.3598 & 68409.8 & 42503.6 \\
\hline 5 & 0.9993 & $\begin{array}{l}0.8634 \\
\end{array}$ & 0.9480 & 0.6522 & 0.3478 & 79306.3 & 48955.4 \\
\hline 6 & 0.9657 & $\mathbf{0 . 8 9 7 9}$ & 0.9408 & 0.6236 & 0.3764 & $\mathbf{5 0 7 5 7 . 5}$ & 32950.2 \\
\hline 7 & 0.8206 & 0.9389 & $\mathbf{0 . 8 6 5 7}$ & 0.6091 & 0.3909 & 55238.2 & 30986.7 \\
\hline 8 & 0.8451 & 0.9767 & 0.8952 & $\mathbf{0 . 5 7 7 7}$ & 0.4223 & 66022.1 & 41756.6 \\
\hline 9 & 0.9315 & 0.9917 & 0.9534 & 0.5849 & 0.4151 & 74758.4 & 49841.8 \\
\hline
\end{tabular}

\footnotetext{
${ }^{50}$ In order to retrieve the unnormalized construction cost index that is used in our hedonic regressions, multiply the $\mathrm{p}_{\mathrm{St}}$ in Table A2 by 19.430 .
} 


\begin{tabular}{|c|c|c|c|c|c|c|c|}
\hline 10 & 0.7932 & 1.0012 & 0.8701 & 0.6028 & 0.3972 & 56354.2 & 29418.2 \\
\hline 11 & 0.8897 & 1.0054 & 0.9323 & 0.5716 & 0.4284 & 43923.9 & 29139.2 \\
\hline 12 & 0.8540 & 0.9963 & 0.9070 & 0.5796 & 0.4204 & 40433.4 & 25140.6 \\
\hline 13 & 0.9089 & 0.9928 & 0.9400 & 0.6091 & 0.3909 & 48019.2 & 28207.9 \\
\hline 14 & 0.8561 & 1.0033 & 0.9111 & 0.5749 & 0.4251 & 45871.6 & 28939.8 \\
\hline 15 & 0.8302 & 0.9934 & 0.8915 & 0.5608 & 0.4392 & 32643.2 & 21362.3 \\
\hline 16 & 0.8275 & 1.0335 & 0.9052 & 0.5768 & 0.4232 & 50301.8 & 29542.7 \\
\hline 17 & 0.8172 & 1.0227 & $\mathbf{0 . 8 9 4 7}$ & 0.5760 & 0.4240 & 55727.6 & 32776.1 \\
\hline 18 & 0.7660 & 1.0761 & 0.8826 & 0.5401 & 0.4599 & 46133.2 & 27964.8 \\
\hline 19 & 0.8222 & 1.0796 & 0.9193 & 0.5688 & 0.4312 & 37023.3 & 21376.5 \\
\hline 20 & 0.8102 & 1.1307 & 0.9312 & 0.5230 & 0.4770 & 65499.6 & 42799.7 \\
\hline 21 & 0.8234 & 1.0808 & 0.9202 & 0.5571 & 0.4429 & 63402.5 & $\mathbf{3 8 3 9 9 . 3}$ \\
\hline 22 & 0.8193 & 1.0166 & 0.8933 & 0.5709 & 0.4291 & 45536.2 & 27586.6 \\
\hline 23 & 0.9182 & 1.0184 & 0.9549 & 0.5844 & 0.4156 & 84597.7 & 54242.0 \\
\hline 24 & 0.9280 & 1.0432 & 0.9704 & 0.5874 & 0.4126 & 78997.1 & 49353.4 \\
\hline 25 & 0.8482 & 1.1313 & 0.9554 & 0.5435 & 0.4565 & 89033.8 & 56073.8 \\
\hline 26 & 0.8647 & 1.1240 & 0.9629 & 0.5684 & 0.4316 & 82155.5 & 47981.8 \\
\hline 27 & 0.8752 & 1.2174 & 1.0049 & 0.5276 & 0.4724 & 54745.4 & 35242.9 \\
\hline 28 & 0.9652 & 1.2003 & 1.0529 & 0.5569 & 0.4431 & 58275.0 & 37288.4 \\
\hline 29 & 1.0077 & 1.1846 & 1.0729 & 0.5841 & 0.4159 & 81411.2 & 49302.1 \\
\hline 30 & 1.0574 & 1.1464 & 1.0894 & 0.6023 & 0.3977 & 75133.8 & 45762.1 \\
\hline 31 & 1.1213 & 1.1818 & 1.1422 & 0.5888 & 0.4112 & \begin{tabular}{|l|l|}
55608.2 \\
\end{tabular} & 36846.5 \\
\hline 32 & 1.0851 & 1.1999 & 1.1273 & 0.5892 & 0.4108 & 76715.5 & 48363.9 \\
\hline 33 & 1.1582 & 1.1922 & 1.1689 & 0.6015 & 0.3985 & 79867.4 & 51405.3 \\
\hline 34 & 1.1906 & 1.2229 & 1.2005 & 0.6031 & 0.3969 & 101378.9 & 64957.9 \\
\hline 35 & 1.0547 & 1.3277 & 1.1594 & 0.5478 & 0.4522 & 81428.1 & 53394.9 \\
\hline 36 & 0.9525 & 1.4341 & 1.1380 & 0.5210 & 0.4790 & 89093.9 & 54399.3 \\
\hline 37 & 0.8510 & 1.3875 & 1.0577 & 0.4874 & 0.5126 & 53469.3 & 34488.5 \\
\hline 38 & 0.8568 & 1.3376 & 1.0421 & 0.5127 & 0.4873 & 68946.2 & 41975.6 \\
\hline 39 & 0.8922 & 1.2744 & 1.0400 & 0.5312 & $\begin{array}{l}0.4688 \\
\end{array}$ & 70170.5 & 43345.1 \\
\hline 40 & 0.9416 & 1.3391 & 1.0953 & 0.5083 & 0.4917 & 56256.6 & 38263.6 \\
\hline 41 & 1.0059 & 1.3753 & 1.1484 & 0.5447 & 0.4553 & 55880.5 & 34160.1 \\
\hline 42 & 1.0844 & 1.3095 & 1.1721 & 0.5729 & 0.4271 & 65109.9 & 40187.5 \\
\hline 43 & 1.1276 & 1.2301 & 1.1684 & 0.5957 & 0.4043 & 74224.9 & 46186.5 \\
\hline 44 & 1.2079 & 1.1293 & 1.1796 & 0.6363 & 0.3637 & 56226.1 & 34379.9 \\
\hline 45 & 1.2352 & 1.1313 & 1.1972 & 0.6316 & 0.3684 & 51188.6 & 32593.4 \\
\hline 46 & 1.2378 & 1.1722 & 1.2152 & 0.6006 & 0.3994 & 66005.2 & 46344.3 \\
\hline 47 & 1.1377 & 1.1709 & 1.1538 & 0.6196 & 0.3804 & 62534.5 & 37307.6 \\
\hline 48 & 1.1335 & 1.1831 & 1.1558 & 0.6051 & $\begin{array}{l}0.3949 \\
\end{array}$ & 114617.4 & 71668.6 \\
\hline 49 & 1.0488 & 1.2317 & 1.1224 & 0.5754 & 0.4246 & 121784.3 & 76512.7 \\
\hline 50 & 1.0656 & 1.2653 & 1.1456 & 0.5920 & 0.4080 & 126806.8 & 73583.2 \\
\hline 51 & 0.9841 & 1.2610 & 1.0919 & 0.5786 & 0.4214 & 134206.6 & 76292.4 \\
\hline 52 & 1.0600 & 1.1391 & 1.0967 & 0.6262 & $\begin{array}{l}0.3738 \\
\end{array}$ & 162863.4 & 90484.8 \\
\hline 53 & 1.0526 & 1.1490 & 1.0953 & 0.6391 & 0.3609 & 129822.9 & 67156.6 \\
\hline 54 & 1.0344 & 1.2650 & 1.1245 & 0.5895 & 0.4105 & 179159.0 & 102028.6 \\
\hline 55 & 0.9894 & 1.4043 & 1.1461 & 0.5586 & 0.4414 & 115655.3 & 64386.7 \\
\hline 56 & 0.9842 & 1.4696 & 1.1657 & 0.5667 & 0.4333 & 161017.5 & 82454.6 \\
\hline 57 & 0.9938 & 1.4558 & 1.1672 & 0.5611 & 0.4389 & 135454.1 & 72318.6 \\
\hline 58 & 1.0273 & 1.4380 & 1.1828 & 0.5636 & 0.4364 & 148467.1 & 82136.8 \\
\hline 59 & 1.0848 & 1.4514 & 1.2249 & 0.5737 & 0.4263 & 113930.3 & 63272.1 \\
\hline 60 & 1.1688 & 1.4531 & 1.2797 & 0.5874 & 0.4126 & 130766.9 & 73881.3 \\
\hline 61 & 1.1090 & 1.4616 & 1.2437 & 0.5910 & 0.4090 & 157941.2 & 82931.5 \\
\hline
\end{tabular}

Table A3 lists the land price indexes for rich, medium and poor wards, $p_{L R t}, p_{L M t}$ and $p_{L P t}$ respectively, the overall land price $\mathrm{p}_{\mathrm{Lt}}{ }^{*}$ and condo sales price $\mathrm{p}_{\mathrm{t}}{ }^{*}$ indexes for each quarter $\mathrm{t}$, that are generated by the hedonic regression model described in section 13. Table A3 also lists the land and structure shares of imputed property values for the section 13 model, defined for quarter $t$ as $\mathrm{s}_{\mathrm{LRt}} \equiv \mathrm{V}_{\mathrm{LRt}} /\left(\mathrm{V}_{\mathrm{Lt}}+\mathrm{V}_{\mathrm{St}}\right), \mathrm{s}_{\mathrm{LMt}} \equiv \mathrm{V}_{\mathrm{LMt}} /\left(\mathrm{V}_{\mathrm{Lt}}+\mathrm{V}_{\mathrm{St}}\right), \mathrm{s}_{\mathrm{LPt}} \equiv \mathrm{V}_{\mathrm{LPt}} /\left(\mathrm{V}_{\mathrm{Lt}}+\right.$ $\left.\mathrm{V}_{\mathrm{St}}\right)$ and $\mathrm{s}_{\mathrm{St}} \equiv \mathrm{V}_{\mathrm{St}} /\left(\mathrm{V}_{\mathrm{Lt}}+\mathrm{V}_{\mathrm{St}}\right)$ where $\mathrm{V}_{\mathrm{Lt}} \equiv \mathrm{V}_{\mathrm{LRt}}+\mathrm{V}_{\mathrm{LMt}}+\mathrm{V}_{\mathrm{LPt}}$ and $\mathrm{V}_{\mathrm{LRt}}, \mathrm{V}_{\mathrm{LMt}}$ and $\mathrm{V}_{\mathrm{LPt}}$ are defined by (39). The sample averages for the 4 shares (with the sample min and max also tabled) were as follows: $0.032 \leq \mathrm{s}_{\mathrm{LRt}} \leq 0.309 ; 0.103 \leq \mathrm{s}_{\mathrm{LMt}} \leq 0.387 ; 0.024 \leq \mathrm{s}_{\mathrm{LPt}} \leq 0.228$; $0.367 \leq \mathrm{s}_{\mathrm{St}} \leq 0.537$. It can be seen that the land shares are extremely unstable, indicating 
that our sample of properties is too small to support the estimation of separate land price indexes for rich, medium and poor wards.

Table A3: Ward Land Prices, $p_{L R t}, p_{L M t}$ and $p_{L P t}$, Overall Land Prices $p_{L t}{ }^{*}$, Overall Condo Prices $p_{t}{ }^{*}$ and Land and Structure Shares of Property Values, $s_{L R t}, s_{L M t}, s_{L P t}$ and $s_{S t}$, for the Section 13 Hedonic Model

\begin{tabular}{|c|c|c|c|c|c|c|c|c|c|}
\hline Quarter & $\mathrm{p}_{\text {LRt }}$ & $\mathbf{p}_{\text {LMt }}$ & $\mathbf{p}_{\mathrm{LPt}}$ & $\mathbf{p}_{\mathbf{L t}}{ }^{*}$ & $\mathbf{p}_{\mathrm{t}}{ }^{*}$ & $\mathbf{S}_{\text {LRt }}$ & $\mathbf{S}_{\text {LMt }}$ & $\mathbf{S}_{\mathbf{L P t}}$ & $\mathbf{S}_{\mathbf{S t}}$ \\
\hline 1 & 1.0000 & 1.0000 & 1.0000 & 1.0000 & 1.0000 & 0.1420 & $\mathbf{0 . 3 2 6 3}$ & 0.0984 & 0.4333 \\
\hline 2 & 0.9469 & 0.8724 & 1.0185 & 0.9263 & 0.9452 & 0.2858 & 0.1820 & 0.1242 & 0.4080 \\
\hline 3 & 0.8774 & 0.8327 & 0.9643 & 0.8739 & 0.8881 & 0.1161 & 0.3870 & 0.0806 & 0.4163 \\
\hline 4 & 0.9095 & 0.9559 & 0.9774 & 0.9531 & 0.9232 & 0.2279 & 0.2495 & 0.1317 & 0.3909 \\
\hline 5 & 0.9420 & $\mathbf{1 . 0 3 2 3}$ & 0.9648 & 0.9934 & 0.9411 & 0.2949 & 0.2191 & 0.1140 & 0.3720 \\
\hline 6 & 0.8586 & 0.9665 & 0.9821 & 0.9320 & 0.9189 & 0.2350 & 0.2759 & 0.0856 & 0.4035 \\
\hline 7 & 0.8507 & 0.7976 & 0.8052 & 0.8234 & 0.8689 & 0.2165 & 0.2398 & 0.1378 & 0.4058 \\
\hline 8 & 0.8544 & 0.8497 & 0.8241 & $\mathbf{0 . 8 5 5 5}$ & 0.9031 & 0.1086 & 0.3384 & 0.1098 & 0.4433 \\
\hline 9 & 0.9231 & 0.9074 & 0.9212 & 0.9255 & 0.9498 & 0.2729 & 0.1721 & 0.1071 & 0.4479 \\
\hline 10 & 0.7659 & 0.7749 & 0.8149 & 0.7834 & 0.8685 & 0.2389 & 0.3075 & $\mathbf{0 . 0 2 4 3}$ & 0.4292 \\
\hline 11 & 0.7000 & 0.8776 & 0.9298 & 0.8120 & 0.8876 & 0.2178 & 0.2538 & 0.0576 & 0.4708 \\
\hline 12 & 1.0170 & 0.8429 & 0.8590 & 0.9076 & 0.9394 & 0.1840 & 0.3167 & 0.0530 & 0.4463 \\
\hline 13 & 0.8931 & 0.8638 & 0.9614 & $\begin{array}{l}0.8838 \\
\end{array}$ & 0.9237 & 0.2466 & 0.2959 & 0.0407 & 0.4168 \\
\hline 14 & 0.8257 & 0.8031 & 0.9320 & 0.8240 & 0.8922 & 0.1895 & 0.2564 & 0.0936 & 0.4605 \\
\hline 15 & 0.7828 & 0.8470 & 0.7996 & 0.7909 & 0.8688 & 0.2681 & 0.1031 & 0.1576 & 0.4712 \\
\hline 16 & 0.8300 & 0.8348 & 0.7936 & 0.8083 & $\begin{array}{l}0.8951 \\
\end{array}$ & 0.2425 & 0.2014 & 0.1119 & 0.4442 \\
\hline 17 & 0.7600 & 0.8179 & 0.8050 & 0.7770 & 0.8718 & 0.2006 & 0.1892 & 0.1504 & $\mathbf{0 . 4 5 9 7}$ \\
\hline 18 & 0.6392 & 0.7649 & 0.8035 & 0.7110 & 0.8523 & 0.1599 & 0.2720 & $\mathbf{0 . 0 8 0 3}$ & 0.4878 \\
\hline 19 & 1.0532 & 0.7805 & 0.8394 & $\mathbf{0 . 8 5 8 7}$ & 0.9441 & 0.2090 & 0.2774 & 0.0673 & 0.4464 \\
\hline 20 & 0.7862 & 0.7738 & 0.8645 & 0.7751 & 0.9149 & 0.1595 & 0.2414 & 0.0909 & $\mathbf{0 . 5 0 8 3}$ \\
\hline 21 & 0.7697 & 0.7564 & 0.8839 & 0.7636 & 0.8881 & 0.2420 & 0.2203 & 0.0687 & 0.4690 \\
\hline 22 & 0.8374 & 0.8086 & 0.7958 & 0.7951 & 0.8821 & 0.1384 & 0.2677 & 0.1301 & 0.4638 \\
\hline 23 & 0.8713 & 0.9202 & 0.9158 & 0.8809 & 0.9335 & 0.2120 & 0.2545 & 0.0826 & 0.4508 \\
\hline 24 & 0.9203 & 0.9128 & 0.9288 & 0.8970 & 0.9531 & 0.1812 & 0.2650 & 0.1168 & 0.4370 \\
\hline 25 & 0.7333 & 0.8906 & 0.8230 & 0.7986 & 0.9294 & 0.1873 & 0.1960 & 0.1279 & 0.4888 \\
\hline 26 & 0.8965 & 0.8739 & 0.8373 & 0.8578 & 0.9621 & 0.2166 & 0.2685 & 0.0551 & 0.4598 \\
\hline 27 & 0.7566 & 0.9402 & 0.8240 & 0.8294 & 0.9820 & 0.1924 & 0.2284 & 0.0846 & 0.4946 \\
\hline 28 & 0.8623 & 1.0644 & 0.8482 & 0.9236 & 1.0316 & 0.1374 & 0.2811 & 0.1191 & 0.4624 \\
\hline 29 & 0.9049 & 1.0934 & 0.8570 & 0.9515 & 1.0422 & 0.1295 & 0.3825 & 0.0428 & 0.4451 \\
\hline 30 & 0.8980 & 1.1015 & 1.0407 & 0.9782 & 1.0438 & 0.1874 & 0.2791 & 0.1111 & 0.4225 \\
\hline 31 & 0.8660 & 1.2466 & 1.0611 & $\mathbf{1 . 0 3 0 4}$ & $\mathbf{1 . 0 8 9 3}$ & 0.1768 & 0.2714 & 0.1133 & 0.4386 \\
\hline 32 & 0.9957 & 1.2283 & 0.9979 & $\mathbf{1 . 0 7 0 7}$ & 1.1204 & 0.2686 & 0.2041 & 0.0834 & 0.4439 \\
\hline 33 & 1.1186 & 1.1980 & 1.0852 & 1.1167 & 1.1443 & 0.1146 & 0.3534 & 0.1144 & 0.4177 \\
\hline 34 & 1.1808 & 1.2333 & 1.1066 & 1.1548 & 1.1793 & 0.1865 & 0.2725 & 0.1202 & 0.4209 \\
\hline 35 & 0.8644 & 1.1356 & 0.9887 & 0.9926 & 1.1270 & 0.1156 & 0.2832 & 0.1155 & 0.4857 \\
\hline 36 & 0.7733 & 0.9415 & 1.0070 & 0.8746 & 1.0994 & 0.1651 & 0.2447 & 0.0743 & 0.5158 \\
\hline 37 & 0.7418 & 0.8688 & 0.8391 & 0.8014 & 1.0366 & 0.1671 & 0.1814 & 0.1144 & 0.5371 \\
\hline 38 & 0.7582 & 0.9504 & 0.7573 & 0.8219 & $\mathbf{1 . 0 2 9 3}$ & 0.1741 & 0.2326 & $\mathbf{0 . 0 8 5 0}$ & 0.5083 \\
\hline 39 & 0.8543 & 0.9359 & 0.8145 & 0.8575 & 1.0257 & 0.1251 & 0.2649 & 0.1033 & 0.5067 \\
\hline 40 & 0.8309 & 1.0456 & 0.8510 & 0.9064 & 1.0809 & 0.1456 & 0.1922 & 0.1346 & 0.5277 \\
\hline 41 & 0.7984 & 1.0235 & 0.9928 & 0.9119 & $\mathbf{1 . 0 9 8 3}$ & 0.3094 & 0.1479 & $\mathbf{0 . 0 5 8 5}$ & 0.4843 \\
\hline 42 & 0.9103 & 1.1981 & 1.0279 & 1.0271 & 1.1441 & 0.1878 & 0.1948 & 0.1686 & 0.4488 \\
\hline 43 & $\mathbf{1 . 0 5 3 7}$ & 1.2094 & 1.0157 & 1.0756 & 1.1424 & 0.1644 & 0.2322 & 0.1744 & 0.4290 \\
\hline 44 & 1.1651 & 1.1548 & 1.2081 & 1.1547 & 1.1510 & 0.2356 & 0.1896 & 0.1930 & 0.3818 \\
\hline 45 & 1.1451 & 1.2703 & 1.1246 & 1.1707 & 1.1616 & 0.0302 & 0.3589 & 0.2276 & 0.3833 \\
\hline 46 & 1.1045 & 1.2861 & 1.2279 & 1.2143 & 1.2043 & 0.0695 & 0.2895 & 0.2224 & 0.4186 \\
\hline 47 & 1.0628 & 1.2114 & 1.0448 & 1.1089 & 1.1409 & 0.1700 & 0.2473 & 0.1874 & 0.3952 \\
\hline 48 & 1.0931 & 1.1953 & 1.0376 & 1.1102 & 1.1464 & 0.1902 & 0.2140 & 0.1891 & 0.4068 \\
\hline 49 & 0.9448 & 1.1040 & 1.0016 & 1.0152 & 1.1077 & 0.2081 & 0.1958 & 0.1525 & 0.4436 \\
\hline 50 & 0.9218 & 1.1066 & 1.0375 & 1.0156 & 1.1210 & 0.2033 & 0.2518 & 0.1175 & 0.4275 \\
\hline 51 & 0.8886 & 1.0214 & 0.9383 & 0.9461 & 1.0752 & 0.1683 & 0.2283 & 0.1642 & 0.4392 \\
\hline 52 & 0.9279 & 1.1346 & 1.0005 & 1.0194 & 1.0764 & 0.2116 & 0.2640 & 0.1292 & 0.3952 \\
\hline 53 & 1.0584 & 1.0737 & 1.0013 & 1.0430 & 1.0953 & 0.2305 & 0.2805 & 0.1224 & 0.3665 \\
\hline 54 & 0.9809 & 1.0544 & 1.0001 & 1.0053 & 1.1126 & 0.2216 & 0.2622 & 0.0925 & 0.4237 \\
\hline 55 & 0.8854 & 0.9593 & 1.0103 & 0.9283 & 1.1140 & 0.2167 & 0.2285 & $\begin{array}{l}0.0997 \\
\end{array}$ & 0.4551 \\
\hline
\end{tabular}




\begin{tabular}{|l|l|l|l|l|l|l|l|l|l|}
\hline $\mathbf{5 6}$ & $\mathbf{0 . 8 1 3 0}$ & $\mathbf{1 . 0 4 5 1}$ & $\mathbf{0 . 9 1 5 7}$ & $\mathbf{0 . 9 2 4 3}$ & $\mathbf{1 . 1 3 4 6}$ & $\mathbf{0 . 1 5 7 5}$ & $\mathbf{0 . 3 0 6 9}$ & $\mathbf{0 . 0 8 9 0}$ & $\mathbf{0 . 4 4 6 5}$ \\
\hline $\mathbf{5 7}$ & $\mathbf{0 . 9 0 2 7}$ & $\mathbf{1 . 0 3 3 7}$ & $\mathbf{0 . 9 3 4 5}$ & $\mathbf{0 . 9 4 9 2}$ & $\mathbf{1 . 1 4 6 4}$ & $\mathbf{0 . 1 5 2 0}$ & $\mathbf{0 . 3 0 7 0}$ & $\mathbf{0 . 0 8 8 1}$ & $\mathbf{0 . 4 5 2 9}$ \\
\hline $\mathbf{5 8}$ & $\mathbf{0 . 8 5 9 0}$ & $\mathbf{1 . 0 5 5 0}$ & $\mathbf{1 . 0 2 3 9}$ & $\mathbf{0 . 9 6 0 4}$ & $\mathbf{1 . 1 4 7 5}$ & $\mathbf{0 . 1 8 6 8}$ & $\mathbf{0 . 2 4 4 1}$ & $\mathbf{0 . 1 1 5 8}$ & $\mathbf{0 . 4 5 3 3}$ \\
\hline $\mathbf{5 9}$ & $\mathbf{1 . 0 0 5 4}$ & $\mathbf{1 . 1 2 0 0}$ & $\mathbf{1 . 0 2 0 1}$ & $\mathbf{1 . 0 3 7 4}$ & $\mathbf{1 . 2 0 2 7}$ & $\mathbf{0 . 1 3 9 3}$ & $\mathbf{0 . 3 3 8 7}$ & $\mathbf{0 . 0 8 8 1}$ & $\mathbf{0 . 4 3 3 9}$ \\
\hline $\mathbf{6 0}$ & $\mathbf{0 . 9 6 0 7}$ & $\mathbf{1 . 2 2 3 8}$ & $\mathbf{1 . 1 4 2 2}$ & $\mathbf{1 . 0 9 4 7}$ & $\mathbf{1 . 2 4 0 8}$ & $\mathbf{0 . 1 9 0 7}$ & $\mathbf{0 . 2 7 9 2}$ & $\mathbf{0 . 1 0 4 4}$ & $\mathbf{0 . 4 2 5 7}$ \\
\hline 61 & $\mathbf{0 . 9 6 6 9}$ & $\mathbf{1 . 1 2 7 1}$ & $\mathbf{1 . 1 2 3 8}$ & $\mathbf{1 . 0 4 7 3}$ & $\mathbf{1 . 2 1 2 6}$ & $\mathbf{0 . 1 4 0 3}$ & $\mathbf{0 . 3 2 3 1}$ & $\mathbf{0 . 1 1 2 9}$ & $\mathbf{0 . 4 2 3 7}$ \\
\hline
\end{tabular}

Table A4: Section 12 Sales Price Index $p_{t}$ and Lowe Index $p_{\text {LowEt, }}$ Traditional Time Dummy Sales Price Index $\mathbf{p}_{\text {TDt }}$ and Quarterly Mean pmeant and Median $\mathbf{p}_{\text {MEDt }}$ Sales Price Indexes

\begin{tabular}{|c|c|c|c|c|c|}
\hline Quarter t & $\mathbf{p}_{\mathrm{t}}$ & p & $\mathbf{p}_{\text {TDt }}$ & p $_{\text {MEANt }}$ & $\mathbf{p}_{\text {MEDt }}$ \\
\hline 1 & 1.0000 & 1.0000 & 1.0000 & 1.0000 & 1.0000 \\
\hline 2 & 0.9708 & 0.9707 & 0.9753 & 0.9759 & 0.9608 \\
\hline 3 & 0.8740 & 0.8740 & 0.9020 & 0.8605 & 0.8898 \\
\hline 4 & 0.9360 & 0.9360 & 0.9256 & 0.9273 & 0.8816 \\
\hline 5 & 0.9480 & 0.9484 & 0.9543 & 0.9559 & 0.9429 \\
\hline 6 & 0.9408 & 0.9403 & 0.9200 & 0.9093 & 0.8429 \\
\hline 7 & 0.8657 & 0.8649 & 0.8763 & 0.8131 & 0.7857 \\
\hline 8 & 0.8952 & 0.8944 & 0.8998 & 0.8634 & 0.8931 \\
\hline 9 & 0.9534 & 0.9541 & 0.9519 & 0.9558 & 0.9747 \\
\hline 10 & 0.8701 & 0.8712 & 0.8877 & 0.8839 & 0.8082 \\
\hline 11 & 0.9323 & 0.9330 & 0.9181 & 0.9961 & 1.0122 \\
\hline 12 & 0.9070 & 0.9073 & 0.9179 & 0.9013 & 0.9935 \\
\hline 13 & 0.9400 & 0.9404 & 0.9328 & 0.9718 & 1.0531 \\
\hline 14 & 0.9111 & 0.9113 & 0.9213 & 0.8667 & 0.8082 \\
\hline 15 & 0.8915 & 0.8913 & 0.9038 & 0.8263 & 0.7714 \\
\hline 16 & 0.9052 & 0.9047 & 0.9360 & 0.8869 & 0.8327 \\
\hline 17 & 0.8947 & 0.8942 & 0.9132 & 0.8638 & 0.8082 \\
\hline 18 & 0.8826 & 0.8822 & 0.8895 & 0.8874 & 0.8490 \\
\hline 19 & 0.9193 & 0.9187 & 0.9194 & 0.9153 & 0.9714 \\
\hline 20 & 0.9312 & 0.9303 & 0.9276 & 0.9977 & 1.0327 \\
\hline 21 & 0.9202 & 0.9199 & 0.9241 & 0.9215 & 0.9306 \\
\hline 22 & 0.8933 & 0.8932 & 0.9210 & 0.9180 & 0.9306 \\
\hline 23 & 0.9549 & 0.9558 & 0.9675 & 1.0054 & 1.0327 \\
\hline 24 & 0.9704 & 0.9711 & 0.9716 & 0.9817 & 1.0122 \\
\hline 25 & 0.9554 & 0.9543 & 0.9715 & 0.9264 & 0.9306 \\
\hline 26 & 0.9629 & 0.9619 & 0.9683 & 0.9831 & 1.0122 \\
\hline 27 & 1.0049 & 1.0034 & 0.9937 & 0.9654 & 0.9714 \\
\hline 28 & 1.0529 & 1.0533 & 1.0453 & 1.0187 & 1.0163 \\
\hline 29 & 1.0729 & 1.0740 & 1.0895 & 1.1508 & 1.1694 \\
\hline 30 & 1.0894 & 1.0908 & 1.0992 & 1.0589 & 1.1347 \\
\hline 31 & 1.1422 & 1.1440 & 1.1298 & 1.1570 & 1.1755 \\
\hline 32 & 1.1273 & 1.1281 & 1.1415 & 1.1823 & 1.1837 \\
\hline 33 & 1.1689 & 1.1710 & 1.1556 & 1.1202 & 1.1347 \\
\hline 34 & 1.2005 & 1.2027 & 1.1892 & 1.1927 & 1.2163 \\
\hline 35 & 1.1594 & 1.1570 & 1.1487 & 1.1420 & 1.1755 \\
\hline 36 & 1.1380 & 1.1329 & 1.1371 & 1.1648 & 1.2163 \\
\hline 37 & 1.0577 & 1.0520 & 1.0694 & 0.9663 & 0.9714 \\
\hline 38 & 1.0421 & 1.0370 & 1.0342 & 0.9852 & 0.9959 \\
\hline 39 & 1.0400 & 1.0354 & 1.0610 & 1.0534 & 1.0939 \\
\hline 40 & 1.0953 & 1.0905 & 1.0962 & 1.1078 & 1.0531 \\
\hline 41 & 1.1484 & 1.1443 & 1.1397 & 1.1274 & 1.1571 \\
\hline 42 & 1.1721 & 1.1687 & 1.1651 & 1.1541 & 1.1776 \\
\hline 43 & 1.1684 & 1.1660 & 1.2010 & 1.1053 & 1.0837 \\
\hline 44 & 1.1796 & 1.1784 & 1.2097 & 1.0496 & 1.0776 \\
\hline 45 & 1.1972 & 1.1963 & 1.1988 & 1.0095 & 1.0122 \\
\hline 46 & 1.2152 & 1.2132 & 1.1911 & 1.0491 & 1.0939 \\
\hline 47 & 1.1538 & 1.1501 & 1.1618 & 1.0504 & 1.0163 \\
\hline 48 & 1.1558 & 1.1521 & 1.1593 & 1.0175 & 1.0531 \\
\hline 49 & 1.1224 & 1.1173 & 1.1330 & 1.0152 & 1.0939 \\
\hline 50 & 1.1456 & 1.1404 & 1.1428 & 1.0561 & 1.0980 \\
\hline 51 & 1.0919 & 1.0879 & 1.1051 & 0.9655 & 0.9714 \\
\hline 52 & 1.0967 & 1.0896 & 1.1046 & 1.0135 & 1.0122 \\
\hline
\end{tabular}




\begin{tabular}{|l|l|l|l|l|l|}
\hline 53 & 1.0953 & 1.0887 & 1.1206 & $\mathbf{0 . 9 5 5 7}$ & $\mathbf{0 . 9 3 2 7}$ \\
\hline 54 & 1.1245 & 1.1208 & 1.1387 & 1.0136 & 1.0143 \\
\hline 55 & 1.1461 & 1.1449 & 1.1496 & 1.0464 & 1.0571 \\
\hline 56 & 1.1657 & 1.1661 & 1.1974 & 1.0377 & 1.0302 \\
\hline 57 & 1.1672 & 1.1669 & 1.1841 & 1.0484 & 1.0551 \\
\hline 58 & 1.1828 & 1.1812 & 1.2061 & 1.0335 & 1.0122 \\
\hline 59 & 1.2249 & 1.2222 & 1.2248 & 1.0726 & 1.0816 \\
\hline 60 & 1.2797 & 1.2753 & 1.2807 & 1.1124 & 1.1347 \\
\hline 61 & 1.2437 & 1.2411 & 1.2733 & 1.1102 & 1.1347 \\
\hline
\end{tabular}

\section{References}

Bostic, R.W., S.D. Longhofer and C.L. Readfearn (2007), "Land Leverage: Decomposing Home Price Dynamics", Real Estate Economics 35:2, 183-2008.

Clapp, J.M. (1980), "The Elasticity of Substitution for Land: The Effects of Measurement Errors", Journal of Urban Economics 8, 255-263.

Court, A. T. (1939), "Hedonic Price Indexes with Automotive Examples", pp. 98-117 in The Dynamics of Automobile Demand, New York: General Motors Corporation.

Diewert, W.E. (1992), "Fisher Ideal Output, Input and Productivity Indexes Revisited", Journal of Productivity Analysis 3, 211-248.

Diewert, W.E. (2007), "The Paris OECD-IMF Workshop on Real Estate Price Indexes: Conclusions and Future Directions", Discussion Paper 07-01, Department of Economics, The University of British Columbia, Vancouver, Canada, V6T 1Z1.

Diewert, W.E. (2010), "Alternative Approaches to Measuring House Price Inflation", Discussion Paper 10-10, Department of Economics, The University of British Columbia, Vancouver, Canada, V6T 1Z1.

Diewert, W.E., J. de Haan and R. Hendriks (2011), “The Decomposition of a House Price Index into Land and Structures Components: A Hedonic Regression Approach", The Valuation Journal 6, (2011), 58-106.

Diewert, W.E., J. de Haan and R. Hendriks (2015), "Hedonic Regressions and the Decomposition of a House Price index into Land and Structure Components", Econometric Reviews, 34:1-2, 106-126.

Diewert, W.E. and C. Shimizu (2015), "Residential Property Price Indices for Tokyo", Macroeconomic Dynamics 19, 1659-1714.

Diewert, W.E. and C. Shimizu (2016), "Alternative Approaches to Commercial Property Price Indexes for Tokyo", Review of Income and Wealth, forthcoming.

Eurostat (2013), Handbook on Residential Property Prices Indices (RPPIs), Luxembourg: Publications Office of the European Union. 
Fisher, I. (1922), The Making of Index Numbers, Boston: Houghton-Mifflin.

Francke, M.K. (2008), “The Hierarchical Trend Model”, pp. 164-180 in Mass Appraisal Methods: An International Perspective for Property Valuers, T. Kauko and M. Damato (eds.), Oxford: Wiley-Blackwell.

Geltner, D. and S. Bokhari (2015), "Commercial Buildings Capital Consumption in the United States", Final Report, MIT Center for Real Estate, November.

Hill, P. (ed.) (2004), Consumer Price Index Manual: Theory and Practice, Geneva: International Labour Office.

Koev, E. and J.M.C. Santos Silva (2008), "Hedonic Methods for Decomposing House Price Indices into Land and Structure Components", unpublished paper, Department of Economics, University of Essex, England, October.

Lowe, J. (1823), The Present State of England in Regard to Agriculture, Trade and Finance, second edition, London: Longman, Hurst, Rees, Orme and Brown.

McMillen, D.P. (2003), "The Return of Centralization to Chicago: Using Repeat Sales to Identify Changes in House Price Distance Gradients", Regional Science and Urban Economics 33, 287-304.

Muth, R.F. (1971), “The Derived Demand for Urban Residential Land”, Urban Studies 8, 243-254.

Rambaldi, A.N., R.R.J McAllister, K. Collins and C.S. Fletcher (2010), "Separating Land from Structure in Property Prices: A Case Study from Brisbane Australia", School of Economics, The University of Queensland, St. Lucia, Queensland 4072, Australia.

Rosen, S. (1974), "Hedonic Prices and Implicit Markets: Product Differentiation in Pure Competition", Journal of Political Economy 82, 34-55.

Schwann, G.M. (1998), "A Real Estate Price Index for Thin Markets”, Journal of Real Estate Finance and Economics 16:3, 269-287.

Shimizu, C., K.G. Nishimura and T. Watanabe (2012), "House Prices from Magazines, Realtors, and the Land Registry", Property Market and Financial Stability, BIS Papers No.64, Bank of International Settlements, March 2012, 29-38.

White, K.J. (2004), Shazam: User's Reference Manual, Version 10, Vancouver, Canada: Northwest Econometrics Ltd. 